OPEN ACCESS

Edited by:

Jinhui Wang,

Center for Cognition and Brain Disorders, Hangzhou Normal University, China

Reviewed by: Gong-Jun Ji,

Anhui Medical University, China Filippo Brighina, Università degli Studi di Palermo, Italy

*Correspondence: Wing Ting To wingting.to@utdallas.edu

Received: 02 November 2017 Accepted: 19 March 2018 Published: 13 April 2018

Citation: To WT, De Ridder D, Hart J Jr. and Vanneste S (2018) Changing Brain Networks Through Non-invasive Neuromodulation.

Front. Hum. Neurosci. 12:128. doi: 10.3389/fnhum.2018.00128

\section{Changing Brain Networks Through Non-invasive Neuromodulation}

\author{
Wing Ting To ${ }^{1 *}$, Dirk De Ridder ${ }^{2}$, John Hart Jr. ${ }^{1}$ and Sven Vanneste ${ }^{1}$ \\ ${ }^{1}$ School of Behavioral and Brain Sciences, The University of Texas at Dallas, Richardson, TX, United States, ${ }^{2}$ Department \\ of Surgical Sciences, Section of Neurosurgery, Dunedin School of Medicine, University of Otago, Dunedin, New Zealand
}

Background/Objective: Non-invasive neuromodulation techniques, such as repetitive Transcranial Magnetic Stimulation (rTMS) and transcranial Direct Current Stimulation (tDCS), have increasingly been investigated for their potential as treatments for neurological and psychiatric disorders. Despite widespread dissemination of these techniques, the underlying therapeutic mechanisms and the ideal stimulation site for a given disorder remain unknown. Increasing evidence support the possibility of non-invasive neuromodulation affecting a brain network rather than just the local stimulation target. In this article, we present evidence in a clinical setting to support the idea that non-invasive neuromodulation changes brain networks.

Method: This article addresses the idea that non-invasive neuromodulation modulates brain networks, rather than just the local stimulation target, using neuromodulation studies in tinnitus and major depression as examples. We present studies that support this hypothesis from different perspectives.

Main Results/Conclusion: Studies stimulating the same brain region, such as the dorsolateral prefrontal cortex (DLPFC), have shown to be effective for several disorders and studies using different stimulation sites for the same disorder have shown similar results. These findings, as well as results from studies investigating brain network connectivity on both macro and micro levels, suggest that non-invasive neuromodulation affects a brain network rather than just the local stimulation site targeted. We propose that non-invasive neuromodulation should be approached from a network perspective and emphasize the therapeutic potential of this approach through the modulation of targeted brain networks.

\footnotetext{
Keywords: transcranial magnetic stimulation, transcranial direct current stimulation, stimulation target, brain hubs, brain networks
}

\section{INTRODUCTION}

The use of invasive and non-invasive neuromodulation for the treatment of neurological and psychiatric disorders has grown exponentially in recent years, with increasing interest in non-invasive neuromodulation. In neurorehabilitation, non-invasive neuromodulation techniques, such as repetitive Transcranial Magnetic Stimulation (rTMS) and transcranial Direct Current Stimulation (tDCS), have been proven to ameliorate symptoms in depressive disorders, pain, aphasia, movement disorders, motor stroke, multiple sclerosis, epilepsy, disorders of consciousness, Alzheimer's disease, tinnitus, schizophrenia, substance abuse, addiction and craving, amongst others (e.g., Fregni et al., 2008; Benninger et al., 2010; Van den Eynde et al., 2010; Zyss, 2010; Freitas et al., 2011; Avenanti et al., 2012; Borckardt et al., 2012; Brunelin et al., 2012; 
Brunoni et al., 2012; Sun et al., 2012; Berlim et al., 2013; Li et al., 2013; Marangolo et al., 2013; Villamar et al., 2013b; Donnell et al., 2015; Douglas et al., 2015; Shekhawat et al., 2016).

RTMS and tDCS are assumed to induce neuroplastic changes through the application of magnetic or electrical stimuli, respectively, directly to a brain area. For rTMS, brief high-current pulses are produced in a coil of wire, called the magnetic coil (Shapira-Lichter et al., 2013). The magnetic field is produced with the line of flux passing perpendicular to the plane of the coil, which is usually placed tangentially to the scalp (ShapiraLichter et al., 2013). RTMS modulates cortical excitability using either inhibitory, low-frequency $(\leq 1 \mathrm{~Hz})$ or facilitatory high-frequency $(\geq 5 \mathrm{~Hz})$ stimulation. Furthermore, there are recently developed rTMS paradigms aimed at modifying cortical excitability, such as theta burst stimulation (TBS), delivered as a continuous (i.e., cTBS) or an intermittent theta burst stimulation (i.e., iTBS) train. cTBS is suggested to be similar to $1 \mathrm{~Hz}$-rTMS, mimicking the long-term depression (LTD) of synaptic plasticity, whereas iTBS is suggested to be excitatory (Di Lazzaro et al., 2005; Huang et al., 2005; Noh et al., 2015). More specifically for cTBS, Ji et al. (2017) have demonstrated that low frequency (LF; $1 \mathrm{~Hz}$ ) rTMS and cTBS share similar short-term aftereffects in both topographical and temporal profiles, suggesting a similar underlying mechanism (Ji et al., 2017). TDCS influences brain excitability by using a low level of continuous electrical current. For tDCS, two (or more) electrodes are placed on the scalp with the current going from the anode to the cathode. As opposed to rTMS, the electrical currents delivered by tDCS are not strong enough to fire an action potential (Radman et al., 2009). The actual plastic effects of both techniques, however, are also dependent on the state of the stimulated cortex. It has been suggested that the stimulation can potentially interact with the prior state of the cortex (Filmer et al., 2014). Several factors can influence excitatory/inhibitory changes of brain stimulations (Filmer et al., 2014), including the state of the brain during stimulation (at rest or paired with a task; Horvath et al., 2014), any intake of substances such as nicotine (Thirugnanasambandam et al., 2011) and even the time of the day (Sale et al., 2007). This sliding of the modification threshold for increased excitation (or long-term potentiation, LTP) and decreased excitation (or LTD), depending on the previous history of neural activity is referred as "metaplasticity" or "homeostatic plasticity" (Abraham and Bear, 1996; Desai, 2003; Lang et al., 2004; Siebner et al., 2004; Abraham, 2008; Cosentino et al., 2012; Hulme et al., 2013; Bocci et al., 2014).

Currently, behavioral manifestations of neurological and psychiatric diseases are seen as a result of alterations in a brain network and its connectivity as opposed to an abnormality in one isolated brain region (Fox et al., 2012b; Shafi et al., 2012; Luft et al., 2014; Fornito and Bullmore, 2015). Neuroscientific research has shifted focus from the properties of individual brain regions to the interactions and connections between brain regions (Fox et al., 2012b). Even in a recent reappraisal of historic cases, such as Phineas Gage, Louis Victor Leborgne, and Henry Gustave Molaison, researchers indicated that the disruptions extended to areas far from the site of the lesions themselves (Thiebaut de Schotten et al., 2015).

Neuromodulation research has also shifted its approach from targeting individual brain regions to targeting entire brain networks (Sale et al., 2015). Increasing evidence points to the influence of neuromodulation on whole brain networks by stimulating just one brain region (or brain hub; Grefkes and Fink, 2011; Fox et al., 2012b). Furthermore, the positive clinical effects of non-invasive neuromodulation in various disorders are presumably caused by the complex interactions between the associated brain network and the stimulation target (Kunze et al., 2016). Interestingly, studies stimulating one important brain hub involved in different processes and disorders, such as the dorsolateral prefrontal cortex (DLPFC), have shown to be effective for several conditions, which is expected since this core hub is involved in general cognitive and emotional processing. On the other hand, studies using different target locations (i.e., various brain areas in the altered brain network) for the same disorder have shown to have similar results, as in the case of tinnitus and major depression. Evidence for the effectiveness of different stimulation targets for one neuropathology as well as evidence for the effectiveness of one stimulation target for different pathologies may indicate an underlying neural network for disorders and may consequently suggest network stimulation as a new stimulation protocol.

In this review we discuss non-invasive neuromodulation techniques, namely rTMS and tDCS and their possible effects on functional connectivity in the brain. We briefly describe the basic brain network models and their hubs. Then, we pursue confirmation for possible functional network effects of rTMS and tDCS across different levels of the central nervous systems, i.e., a whole-brain functional connectivity level as well as a neurometabolite concentration level. Lastly, we present further evidence in clinical studies demonstrating possible effects on the functional connectivity of the brain for rTMS and tDCS on different stimulation sites and different disorders by: (1) presenting various effective stimulation sites for one disorder; and (2) presenting one effective stimulation site for different disorders.

\section{BRAIN NETWORKS AND THEIR HUBS}

The human brain is a complex network of interlinked regions (van den Heuvel and Sporns, 2011; Fornito et al., 2013). This network can be mathematically represented as graphs, comprised of nodes (neuronal elements) and edges (their connections) that describe the brain's structural connectivity (Fornito et al., 2013; van den Heuvel and Sporns, 2013a). A subset of nodes (brain regions) with strong internal interaction and relatively weak external associations are represented as modules (van den Heuvel and Sporns, 2013b; Cocchi et al., 2015). Communication between modules is supported by brain hubs, which are regions that are densly interconnecteds with other brain areas and play an important role in the integration of information between different parts of the network (van den Heuvel and Sporns, 2011; Cocchi et al., 2015; see Figure 1). 


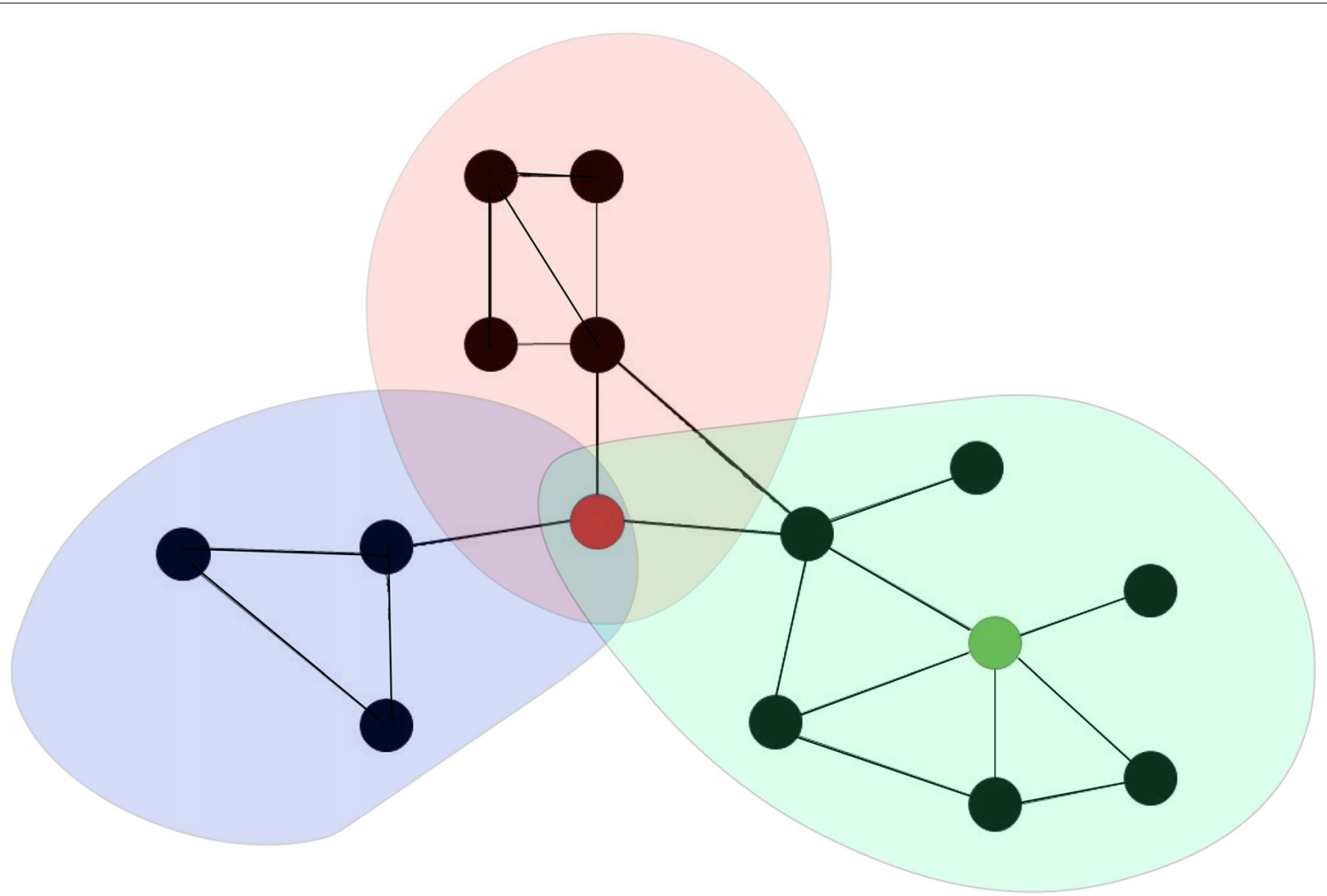

FIGURE 1 | Brain networks can be represented as a graph comprising of a set of nodes (black dots) and a collection of edges (black lines in between the black dots). A subset of nodes of the network that show strong interaction among each other than other nodes in other subset of nodes are represented as modules (colored communities). Provincial hubs are highly connected nodes that primary connect nodes in the same module (e.g., green node). Connector hubs are highly connected nodes that has a diverse connectivity profile because it is connecting to several different modules within the brain network (e.g., red node).

In addition to the classification of hubs on the basis of anatomical or structural connectivity, numerous studies have also investigated the existence of "functional hubs" derived from networks of dynamic interactions between brain regions (van den Heuvel and Sporns, 2013a). Connectivity in brain networks can indeed be assessed in different ways (Friston, 2011). Anatomical or structural connectivity, the stable direct physical pathways linking spatially distinct brain areas, is distinguished from dynamic functional connectivity and effective connectivity (Friston, 2011; Shafi et al., 2012; Fornito et al., 2013; Luft et al., 2014). Effective connectivity refers to the directional flow of information or the causal relationships between (Friston, 2011; Shafi et al., 2012; Fornito et al., 2013; Luft et al., 2014) nodes, whereas functional connectivity simply computes measures of statistical independence (correlation) between nodes (Friston, 2011; Shafi et al., 2012; Fornito et al., 2013; Luft et al., 2014).

Conceptualizing the brain as a network (Sporns et al., 2005) might have important implications for understanding clinical brain disorders (Bullmore and Sporns, 2012; Menon, 2013; Rubinov and Bullmore, 2013; van den Heuvel and Sporns, 2013a; Crossley et al., 2014). A generic property of a network is that dysfunction can spread easily between linked elements, leading to pathological cascades that include large parts of the system (Buldyrev et al., 2010; Huang et al., 2011). Fornito et al. (2015) have proposed different versions of a maladaptive response that can mediate the spread of pathology throughout the connectome, as well as the resources and processes that enable adaptation. Studies have suggested that damage to "provincial" hubs-those that are the primary link to other nodes in the same module and have an important role in functional specialization-will lead to specific clinical deficits, whereas damage to "connector" hubs-those that have links that are distributed across multiple different modules and have a central role in functional integration-will result in more complex and pervasive dysfunction and is proposed to impair multiple behavioral domains (Fornito et al., 2015). In general, it has been suggested that brain hubs are indeed involved in the anatomy of various brain disorders (Crossley et al., 2014). Studies have further found extensive anatomical overlap between structural abnormalities or lesions in various brain disorders (Crossley et al., 2014), with a similar result reported in another study of a subgroup of psychiatric disorders (Goodkind et al., 2015). For example, a recent meta-analysis of structural and functional neuroimaging studies found a collective core of brain regions affected by most psychiatric disorders, centered on dorsal anterior cingulate cortex (dACC) and the insula (Goodkind et al., 2015; Downar et al., 2016). These nodes correspond to an anterior cingulo-insular or "salience" network and are suggested to stand at a crossroads 
within the functional architecture of the brain, acting as a switch to deploy other major functional networks according to motivational demands and environmental constraints (Downar et al., 2016). The authors proposed these regions as promising targets for brain stimulation in psychiatric disorders. With more advanced designs of non-invasive neuromodulation, studies have shown the possibility of targeting these deeper brain structures using, for example, double cone coil rTMS (Hayward et al., 2007; De Ridder et al., 2011; Deng et al., 2014; Kreuzer et al., 2015) or high definition tDCS (DaSilva et al., 2015).

\section{INFLUENCE OF NON-INVASIVE NEUROMODULATION IN FUNCTIONAL BRAIN NETWORKS}

The effects of non-invasive neuromodulation on networkbased connectivity can be observed and investigated across different levels of the central nervous system, such as a whole-brain functional connectivity level as well as a neurometabolite concentration level. In this section, studies adopting both a "macro" as well as a "micro" perspective of the brain's neural structure will be examined to understand the distributed processing of neuromodulation in functional brain networks.

\section{MACRO-Level: Assessing Network Connectivity With Task-Independent and Task-Related Neuroimaging}

Recent studies have shown that non-invasive neuromodulation techniques affect brain connectivity patterns both while performing tasks and while at rest (Grefkes et al., 2010; Alon et al., 2011; Keeser et al., 2011a; Polanía et al., 2011a, 2012; Vanneste and De Ridder, 2011; Meinzer et al., 2012, 2013; Peña-Gómez et al., 2012; Chib et al., 2013; Park et al., 2013; Stagg et al., 2013; Ding et al., 2014; Weber et al., 2014), suggesting that stimulation is not influencing the target area in isolation, but rather a brain network (Alon et al., 2011). These techniques enable the perturbation of large-scale neural systems and may facilitate in testing whole brain models (Breakspear, 2017). Most studies examined the effects of tDCS or rTMS on brain connectivity by analyzing changes in functional networks during task-independent (resting-state) or task-related neuroimaging (e.g., EEG, fMRI). Therefore, in this article, only studies combining neuroimaging with tDCS and rTMS will be presented.

Task-independent or resting-state neuroimaging studies have investigated the effect of tDCS or rTMS on short term reorganization in functional networks in healthy subjects (for review see Luft et al., 2014) and in patients (e.g., tinnitus patients, Vanneste and De Ridder, 2011) using fMRI (for review see Luft et al., 2014) or EEG (Polanía et al., 2011a; Vanneste and De Ridder, 2011). The studies varied in how they defined the nodes of the network (Luft et al., 2014). Most studies identified specific regions of interest to look into brain connectivity.
For tDCS studies, the specific brain regions of interest varied from M1 (Polanía et al., 2011a,b, 2012) to DLPFC (Vanneste and De Ridder, 2011; Park et al., 2013; Stagg et al., 2013; Weber et al., 2014). These studies all combined tDCS with either resting-state EEG or resting-state fMRI to examine for example whether tDCS induces increased or decreased functional connectivity between specific brain areas (e.g., intrahemispheric or interhemispheric connectivity changes; e.g., Polanía et al., 2011b; Sehm et al., 2013) or whether tDCS modulates functional connectivity of certain circuits (e.g., Alon et al., 2011; Polanía et al., 2012) in a non-clinical population. For instance, studies using tDCS to target the left DLPFC in healthy subjects have been shown to influence regional electrical activity in both surface level and deeper structures using resting-state EEG (Keeser et al., 2011b) and resting-state fMRI (Keeser et al., 2011a; Peña-Gómez et al., 2012; Park et al., 2013; Stagg et al., 2013). Keeser et al. (2011b) used resting-state EEG and sLORETA to demonstrate that tDCS on the left DLPFC influences electrical activity in both during surface areas measured by EEG and deeper structures in the prefrontal lobe, such as the medial frontal gyrus, the anterior cingulate cortex and the subgenual anterior cingulate cortex. They further suggested that tDCS might influence this whole network during resting state, making it easier to activate the network during consecutive cognitive task performances, as a positive impact of tDCS was seen on a following n-back task (on error rate, accuracy and reaction time). Park et al. (2013) used resting-state fMRI to demonstrate that tDCS on the left DLPFC increased interhemispheric connectivity, suggesting that this mechanism may enhance cognitive functioning. Using restingstate fMRI, Keeser et al. (2011a) further assessed resting-state connectivity after left DLPFC and found significant changes in the default mode network (DMN) and the right and left frontal parietal network (FPN), both close to the primary stimulation site and in connected brain areas. In addition, Peña-Gómez et al. (2012) revealed increased synchrony in the anticorrelated network-a network of brain areas revealing strong negative activity correlation with the DMN and associated with cognitive processing-components and reduced synchrony in the DMN after tDCS over the left DLFPC, assessed with resting-state fMRI. This finding suggests that tDCS over the DLPFC may enhance the flexible balance between brain networks by enhancing network connectivity for cognitive demands while reducing DMN activity. Interestingly, Stagg et al. (2013) focused on brain perfusion changes during left DLPFC tDCS (using wholebrain arterial spin labeling) and found decreased functional connectivity between the left DLPFC and the bilateral thalami after tDCS, possibly offering a mechanistic explanation for the analgesic effects of tDCS in pain studies while adding weight to the hypothesis that the DLPFC modulates pain via a decrease in thalamic activity. In patient populations, one study in tinnitus patients, combining tDCS with resting-state EEG, had demonstrated that bifrontal DLPFC tDCS was able to suppress tinnitus by modulating the pregenual anterior cingulate cortex, the parahippocampal area, and the right primary auditory cortex in resting-state spontaneous brain activity (Vanneste and De Ridder, 2011). This study is the first conducted in a 
patient population to provide support that $\mathrm{tDCS}$ has an impact not only on the underlying DLPFC, but also indirectly on functionally connected brain areas relevant for tinnitus distress and tinnitus intensity in tinnitus patients. For rTMS studies, more specifically theta burst transcranial stimulation (TBS), only a few resting-state studies have explored whether TBS would lead to changes in resting-state functional connectivity in healthy subjects. The specific brain regions of interest encompassed a broad range including: (1) the occipital cortex (Rahnev et al., 2013); (2) anterior insula/frontal operculum (Gratton et al., 2013); (3) the primary somatosensory cortex (Valchev et al., 2015); (4) the primary motor cortex (CárdenasMorales et al., 2014; Cocchi et al., 2015; Noh et al., 2015); (5) the precuneus (Mancini et al., 2017); and (6) the DLPFC (Gratton et al., 2013; Mastropasqua et al., 2014; Iwabuchi et al., 2017). These studies all combined TBS with either resting-state EEG or resting-state fMRI to investigate whether TBS can induce increased or decreased functional connectivity between specific brain areas (e.g., intrahemispheric or interhemispheric connectivity changes; e.g., Rahnev et al., 2013; Noh et al., 2015; Valchev et al., 2015; Mancini et al., 2017) in healthy subjects. For example, studies targeting the left DLPFC in healthy subjects using TBS have demonstrated influence on regional and more remote functions using resting-state fMRI (e.g., Gratton et al., 2013; Iwabuchi et al., 2017). Gratton et al. (2013) demonstrated that left DLPFC cTBS increased connectivity with regions in the frontal, parietal, and cingulate cortices, suggesting that acute disruption by TBS to cognitive control regions can cause widespread changes in network connectivity not limited to the targeted networks. Iwabuchi et al. (2017) further explored whether prefrontal iTBS targeting the left DLPFC can modulate crucial limbic structures such as the insula, which can explain the therapeutic effects of DLPFC rTMS in depression. They have found that iTBS significantly dampened fronto-insular connectivity, demonstrating that left DLPF iTBS was able to modulate the right anterior insula (Iwabuchi et al., 2017).

Task-related neuroimaging studies have also examined short-term reorganization in healthy subjects (e.g., O'Shea et al., 2007; Andoh and Paus, 2011; Chib et al., 2013; Weber et al., 2014) after non-invasive neuromodulation. These studies all combined non-invasive neuromodulation such as tDCS or rTMS with neuroimaging such as EEG or fMRI, while performing a task, allowing for more process-specific findings. These studies demonstrate that non-invasive neuromodulation can modulate specific task functions, regions, and network interactions. Studies differ in the task performed, the neuromodulation technique used (e.g., tDCS or rTMS), the neuroimaging technique used (e.g., EEG or fMRI), and the specific brain region of interest. For example, Andoh and Paus (2011) combined off-line $10 \mathrm{~Hz}$ rTMS targeting the left and right posterior temporal area of Wernicke (LTMP; performed outside the magnetic resonance scanner) with $\mathrm{AMRI}$, acquired during the performance of a word recognition task. Following the hypothesis that some brain functions operate in a state of interhemispheric compensation (i.e., recruiting homologous regions in the contralateral hemisphere) to recover after a virtual lesion
(O'Shea et al., 2007), Andoh and Paus (2011) hypothesized that rTMS applied over the LTMP area will induce changes in the task-related fMRI response both locally and distally, namely in the homologous area in the contralateral hemisphere (RTMP). Similarly, they predicted that rTMS applied over the RTMP area would induce changes in the task-related fMRI response in the contralateral hemisphere (LTMP). Their result showed that rTMS increased task-related fMRI response in the homolog areas contralateral to the stimulated areas, consistent with the hypothesis regarding the role of homolog areas in the contralateral hemisphere for preserving behavior after neural interference. Interestingly, studies have also combined non-invasive neuromodulation with simultaneous task-based neuroimaging (e.g., Meinzer et al., 2012, 2013). For example, Meinzer and Colleagues have used fMRI during overt semantic word generation and simultaneous intrascanner anodal tDCS on the left inferior frontal gyrus (IFG) to investigate its effects on performance and task-related activity in healthy young adults (Meinzer et al., 2012) and in healthy older adults (Meinzer et al., 2013). The study in healthy young adults showed improved word-retrieval during anodal tDCS paralleled by selectively reduced task-related activation in the left ventral IFG, indicative of more efficient neural processing. In healthy older adults, anodal tDCS significantly improved performance up to the level of the younger controls and task-related fMRI showed reduced task-related hyperactivity in the bilateral prefrontal cortices, the anterior cingulate gyrus, and the precuneus (brain areas that were "hyperactive" in older compared to younger adults). Thus, this study showed that one single session of tDCS can temporarily reverse some age-associated changes in brain activity and connectivity.

\section{MICRO-Level: Assessing Network Connectivity With Chemical Markers of Neural Plasticity/Neurometabolites}

While the induced effects of non-invasive neuromodulation have been investigated using neuroimaging techniques that provide information of the brain's neural structure on a "macro" network-based connectivity level, tDCS studies have also been examining the effects of the brain's neural structure on a "micro" neurometabolites level, in an attempt to explain local and distributed processing in functional brain networks (Hunter et al., 2013). TDCS has been known to influence neurophysiological mechanisms responsible for neuroplasticity by modulating the excitability of glutamatergic pyramidal neurons in the underlying cortex (Radman et al., 2009). These mechanisms involve the potentiation of synaptic glutamatergic receptors (Liebetanz et al., 2002; Nitsche et al., 2005) and decreased neurotransmission of GABA (Nitsche et al., 2004; Stagg et al., 2009; Stagg and Nitsche, 2011; Hunter et al., 2015; for a review, see Medeiros et al., 2012). Since there is evidence that the sum of glutamate and glutamine (Glx) levels are increased by tDCS (Clark et al., 2011) and that there is a strong relationship between Glx and functional connectivity (Horn et al., 2010; Kapogiannis et al., 2013), 
studies have been investigating whether tDCS-evoked changes in Glx may predict variations in functional connectivity within and between both local and distributed brain networks (e.g., Hunter et al., 2015). Hunter et al. (2015) found evidence that the after-effects of right parietal tDCS on glutamatergic signaling and network connectivity contribute to local, crosshemispheric, and subcortical alterations. They found increases in baseline glutamatergic signaling that originate from the parietal site of the stimulation, with the precuneus possibly acting as an intermediate node that modulates glutamatergic signaling in other pathways that include the bilateral superior parietal lobule, ACC, salience, left frontal-parietal network, and the basal ganglia networks. They further suggest that cross-hemispheric connectivity, specifically for the bilateral inferior parietal network, may be more readily influenced by other neurotransmitter pathways, such as GABA. Recently, studies have been conducted to investigate the involvement of GABAergic signaling pathways that may influence network connectivity. Stagg et al. (2014) showed that tDCS on M1 decreased GABA levels within M1 and increased resting motor network connectivity. They suggested that the networklevel connectivity within the motor system is related to the degree of inhibition in M1, a major node within the motor network. Bachtiar et al. (2015) replicated this tDCS experiment and confirmed the decreased GABA levels within M1 and increased resting motor network connectivity. However, they did not find a relationship between the change in GABA levels in M1 and the change in functional connectivity, suggesting that it might be driven by distinct underlying mechanisms.

\section{VARIOUS STIMULATION TARGETS FOR A SPECIFIC DISORDER}

Non-invasive neuromodulation studies targeting different brain areas to treat one specific disorder have shown to have similar positive results. In this section, we provide support to demonstrate that rTMS and tDCS targeting different nodes of the dysfunctional brain network of a specific disorder can have similar positive effects, using tinnitus and depression as illustrative examples. See Tables 1, 2 for a summary of non-invasive brain stimulation (NIBS) targets for tinnitus and depression.

\section{Tinnitus}

Tinnitus is a common and distressing disorder that is characterized by the perceived sensation of a sound in the absence of an external sound source (Langguth et al., 2012; De Ridder et al., 2014). Like other disorders, tinnitus can

TABLE 1 | Non-invasive brain stimulation (NIBS) targets in tinnitus.

\section{Stimulation target}

Repetitive transcranial magnetic stimulation

HF rTMS to the left auditory cortex

LF rTMS to the left auditory cortex

LF to the right DLPFC

Combined modulation of HF left DLPFC then LF left temporal cortex

Combined modulation of HF left DLPFC then LF left and right temporoparietal cortex

Transcranial direct current stimulation

Anode left auditory cortex-cathode contralateral supraorbital region

Anode right DLPFC - cathode right DLPFC

Simultaneuos 1 anode prefrontal cortex -2 cathode left and right auditory cortex

\section{References}

e.g., Plewnia et al. (2003) and Fregni et al. (2006)

e.g., Eichhammer et al. (2003) and Langguth et al. (2003)

e.g., De Ridder et al. (2013)

e.g., Kleinjung et al. (2008)

e.g., Lehner et al. (2013)

e.g., Fregni et al. (2006), Garin et al. (2011), Shekhawat et al. (2013) and Forogh et al. (2016)

e.g., Vanneste et al. (2010), Vanneste and De Ridder (2011),

De Ridder and Vanneste (2012), Faber et al. (2012) and Frank et al. (2012) e.g., Pal et al. (2015)

HF, high frequency; rTMS, repetitive Transcranial Magnetic Stimulation; LF, low frequency; DLPFC, dorsolateral prefrontal cortex.

TABLE 2 | NIBS targets in depression.

\section{Stimulation target}

Repetitive transcranial magnetic stimulation

HF rTMS to left DLPFC

LF rTMS to the right DLPFC

Combined modulation of HF left DLPFC then LF right DLPFC during same session

Combined modulation of HF left than right DMPFC

Transcranial direct current stimulation

Anode left DLPFC - cathode right supraorbital region

Anode left DLPFC-cathode right DLPFC
References

e.g., Pascual-Leone et al. (1996), Berman et al. (2000), Rossini et al. (2005),

Loo et al. (2007), Bretlau et al. (2008), George et al. (2010) and

Baeken et al. $(2013,2014)$

e.g., Klein et al. (1999), Januel et al. (2006), Fitzgerald et al. (2008),

Bares et al. (2009) and Aguirre et al. (2011)

e.g., Hausmann et al. (2004), Fitzgerald et al. (2006a, 2012), Garcia-Toro et al. (2006),

McDonald et al. (2006), Pallanti et al. (2010) and Blumberger et al. (2012a)

e.g., Downar et al. (2014), Salomons et al. (2014) and Bakker et al. (2015)

e.g., Boggio et al. (2008a,b), Loo et al. (2012), Palm et al. (2012) and

Bennabi et al. (2015)

e.g., Ferrucci et al. (2009), Brunoni et al. (2013, 2014),

Valiengo et al. $(2013,2016,2017)$

HF, high frequency; rTMS, repetitive Transcranial Magnetic Stimulation; DLPFC, dorsolateral prefrontal cortex; LF, low frequency; DMPFC, dorsomedial prefrontal cortex. 
be perceived as a phenomenological unified coherent percept, binding separable clinical characteristics, such as the tinnitus loudness, the tinnitus sidedness, the tinnitus type (pure, tone, noise), the associated distress and so on (De Ridder et al., 2014). Based on neuroimaging studies, a group of tinnitus researchers have proposed a tinnitus brain model consisting of multiple parallel, dynamic, and partially overlapping subnetworks each representing a specific aspect of tinnitus (see De Ridder et al., 2014). However, the revealed subnetworks encoding the different aspects of the tinnitus percept, e.g., the distress network, can be similar to other pathologies, such as pain (Moisset and Bouhassira, 2007; De Ridder et al., 2013, 2014). Communication between these different subnetworks is proposed to occur at brain hubs, brain areas that are involved in multiple subnetworks simultaneously (De Ridder et al., 2014).

In non-invasive neuromodulation studies, researchers mostly targeted the left auditory cortex (temporal or temporoparietal cortical areas), but some have investigated the DLPFC as a target both in isolation and in a multisite stimulation approach, to suppress tinnitus. The left auditory cortex and the DLFPC are believed to be part of a neural network that appears to play a significant role in tinnitus perception (Shekhawat et al., 2016). The auditory cortex has become a common stimulation target for tinnitus, as past neuroimaging studies have shown over-activation of the left auditory cortex in tinnitus patients (Arnold et al., 1996; Lockwood et al., 1998). Repetitive TMS (Fregni et al., 2006), as well as anodal tDCS to the auditory cortex (left temporal or temporoparietal cortices areas; Fregni et al., 2006; Garin et al., 2011), were found to suppress tinnitus. For TMS, LF and high frequency (HF) were investigated to interrupt the tinnitus percept. The first rTMS case studies (shamcontrolled) in tinnitus mostly investigated LF rTMS targeting the left auditory cortex in an attempt to inhibit the hyperactivity in this area (Eichhammer et al., 2003; Langguth et al., 2003). Eichhammer et al. (2003) found considerable improvement in tinnitus in two out of three patients after 1 week (five consecutive days) with LF (1 Hz) rTMS with 2000 stimuli per day, measured by the Tinnitus Questionnaire (assessing different tinnitus complaints e.g., distress, auditory perceptual difficulties, sleep disturbance; Eichhammer et al., 2003). Langguth et al. (2003) described a 4-week case study involving LF $(1 \mathrm{~Hz})$ rTMS treatment ( 5 days of treatment per week with 2000 stimuli/day) targeting the left auditory cortex. This treatment resulted in remarkable effects, enduring for several weeks, measured by the Tinnitus Questionnaire (assessing different tinnitus complaints e.g., distress, auditory perceptual difficulties, sleep disturbance) and paralleled by altered cortical excitability. Later on, HF rTMS was applied to the left auditory cortex with the belief that a virtual temporary lesion of this area can induce a transient reduction in tinnitus (Plewnia et al., 2003). Plewnia et al. (2003) applied HF $(10 \mathrm{~Hz})$ via five stimulation trains at different scalp positions in 14 tinnitus patients and found only a significant reduction in tinnitus when targeting the left temporal and left temporoparietal cortex (targeting the DLPFC did not yield in significant results) using a self-rating tinnitus reduction scale. Fregni et al. (2006) replicated the findings of Plewnia et al. (2003) applying $\mathrm{HF}(10 \mathrm{~Hz})$ via nine stimulation trains of 30 stimuli and found transient tinnitus reduction, measured by the same self-rating tinnitus reduction scale, as well as a self-rating tinnitus intensity scale (Fregni et al., 2006). Moreover, they tested whether tDCS targeting the left auditory cortex would yield in similar effects. Their results showed that even $3 \mathrm{~min}$ of $1 \mathrm{~mA}$ anodal tDCS targeting the left auditory cortex induces similar transient tinnitus reduction as with HF rTMS (Fregni et al., 2006). TDCS, as well as other transcranial electrical stimulation (tES) techniques, such as transcranial random noise stimulation (tRNS) and transcranial alternating current stimulation (tACS), targeting the left auditory cortex has been further investigated by other researchers. Garin et al. (2011) showed that a single session 20-min session of $1 \mathrm{~mA}$ anodal tDCS significantly reduced tinnitus intensity, as measured by a Visual Analogue Scale. Although, Shekhawat et al. (2013) have demonstrated that $2 \mathrm{~mA}$ anodal tDCS for 20 min targeting the left auditory cortex was the most effective stimulation parameter for one session (measured by the Clinical Global Improvement measure and the Tinnitus Loudness Visual Analogue Scale), Forogh et al. (2016) did not find any significant effects when they applied the same for five sessions in patients with tinnitus (measured by the Clinical Global Improvement measure, the Tinnitus loudness Visual Analogue Scale, Tinnitus related-distress Visual Analogue Scale, and the Tinnitus Handicap Inventory). Interestingly, electrical stimulation where the stimulation current is varied randomly, i.e., tRNS was found to be superior both to tDCS (where stimulation current is held constant) and tACS (where stimulation current is time dependent with a sinusoidal shape) when applied bilaterally over the temporal cortex (Vanneste et al., 2013a; Van Doren et al., 2014). More recently, the introduction of High-Definition tDCS (HD-tDCS) has improved the spatial accuracy of conventional tDCS by using arrays of smaller "highdefinition" electrodes instead of two large pad electrodes (Datta et al., 2009; Dmochowski et al., 2011; Guleyupoglu et al., 2013; Villamar et al., 2013a; Heimrath et al., 2015; Shekhawat et al., 2016). Shekhawat et al. (2016) have investigated the effect of stimulation location (left auditory cortex or DLPFC), stimulation duration (10 $\mathrm{min}$ or $20 \mathrm{~min}$ ), and stimulation intensity (center anode $1 \mathrm{~mA}$ or $2 \mathrm{~mA}$ ) on tinnitus loudness and annoyance using $4 \times 1$ HD-tDCS (Shekhawat et al., 2016). They concluded that a higher intensity $(2 \mathrm{~mA})$ and a longer duration $(20 \mathrm{~min})$ of stimulation were more effective, but either stimulation location was equally effective for suppressing tinnitus loudness and annoyance (Shekhawat et al., 2016).

The other common stimulation target investigated for transient tinnitus suppression is the DLPFC. The prefrontal cortex is believed to play a vital role in tinnitus, since it is critically involved in the integration of sensory and emotional aspects of tinnitus, as first mentioned by Jastreboff (1990; Kleinjung et al., 2008) and confirmed by recent studies as an essential part of the tinnitus distress network (Vanneste et al., 2010). Additionally, electrophysiological studies suggest that tinnitus occurs as a result of dysfunctional top-down inhibitory mechanisms originating in the prefrontal lobe (Norena et al., 1999), indicating that the prefrontal cortex does not only color tinnitus perception, but may efficiently switch the perceived 
signal on and off (Rauschecker et al., 2010; Frank et al., 2012), therefore being able to influence both tinnitus distress and intensity. Bifrontal tDCS where the anode is placed over the right DLPFC and the cathode over the left DLPFC, has been investigated in several tinnitus studies (Vanneste et al., 2010; Vanneste and De Ridder, 2011; De Ridder and Vanneste, 2012). The first clinical study conducted in 478 tinnitus patients by Vanneste et al. (2010) revealed that one 20-min session of $1.5 \mathrm{~mA}$ tDCS with a right anode and left cathode set up (and not left anode, right cathode) modulated tinnitus perception in $29.9 \%$ of the tinnitus patients. For these responders, a significant reduction was found for both tinnitus intensity and tinnitus-related distress (Vanneste et al., 2010). These results were further confirmed in a follow up study in which resting-state EEG was added before and after tDCS to unravel the mechanism by which tDCS suppresses tinnitus (Vanneste and De Ridder, 2011). This study provides evidence that bifrontal tDCS can suppress tinnitus intensity and tinnitus-related distress by modulating the pregenual ACC, the parahippocampal area, and the right primary auditory cortex in resting-state spontaneous brain activity (Vanneste and De Ridder, 2011). Furthermore, the study by De Ridder and Vanneste (2012) also supports bifrontal tDCS as a technique to reduce tinnitus intensity and distress, as one session of bifrontal tDCS elicited a larger reduction in tinnitus intensity and distress compared to EEG driven tDCS. TACS targeting the DLPFC has also been investigated (Vanneste et al., 2013b), as it could theoretically normalize alpha power, which is known to decrease in tinnitus (Lorenz et al., 2009). However, tACS did not modulate tinnitus loudness and annoyance like bifrontal tDCS was able to (Vanneste et al., 2013b). Therefore, repetitive sessions of bifrontal tDCS was investigated as a potential treatment (De Ridder and Vanneste, 2012; Faber et al., 2012; Frank et al., 2012). Frank et al. (2012) found that six 30-min sessions of $1.5 \mathrm{~mA}$ tDCS (right anode and left cathode) only resulted in a small clinical impact on tinnitus loudness and discomfort. Faber et al. (2012) investigated six sessions of tDCS for left and right anodal DLPFC and found that both active conditions (irrespective of the anodal position) were able to reduce tinnitus annoyance but not tinnitus intensity. For magnetic stimulation, LF $(1 \mathrm{~Hz})$ rTMS on the right DLPFC also resulted in a reduction in the perceived loudness of tinnitus after one session (200 pulses), as well as after 10 sessions of rTMS ( 600 pulses), which is mediated by the functional connections between the DLPFC, and a network consisting of the ACC, the parahippocampus, and the auditory cortex (De Ridder et al., 2013).

An increasing amount of studies are investigating the stimulation the two common stimulation targets in tinnitus patients, the auditory cortex and the DLPFC, simultaneously or one stimulation after the other. One tDCS study simultaneously targeted the auditory cortex with the DLPFC by placing the cathode on the auditory cortex and the anode over the prefrontal cortex for five sessions (Pal et al., 2015). However, this stimulation set up did not yield in an improvement in any tinnitus measures (Pal et al., 2015). Two studies suggest that the efficacy of rTMS in tinnitus can be enhanced by stimulating prefrontal cortical areas in addition to the auditory cortex (Kleinjung et al., 2008; Lehner et al., 2013). A study by Kleinjung et al. (2008) combined HF prefrontal and LF temporal rTMS. Patients received either LF temporal rTMS or a combination of HF prefrontal and LF temporal rTMS. Directly after therapy they found an improvement for both groups, but no differences between the groups. An evaluation after 3 months revealed a remarkable benefit from the use of combined prefrontal and temporal rTMS treatment. These findings suggest that auditory and non-auditory brain areas are involved in tinnitus psychopathology. Another study by Lehner et al. (2013) combined HF prefrontal with LF left and right temporal rTMS (left DLPFC, left temporoparietal cortex, and right temporoparietal cortex). Patients received either LF temporal rTMS or a combination of HF prefrontal and LF temporal rTMS. They found that multisite rTMS is significantly superior to temporal rTMS and represents a promising strategy for enhancing treatment effects of rTMS in tinnitus. Several authors have (e.g., Vanneste and De Ridder, 2012) already stated that the perception of tinnitus involves large and complex interconnected networks and that tinnitus can be a result of a dysfunction in any part of the system. Thus, modulation of any part of this network may interfere with tinnitus perception (Vanneste and De Ridder, 2012). However, stimulating the auditory cortex alone might not be sufficient to achieve long lasting improvement of tinnitus severity (Lehner et al., 2013). Accordingly, the idea of network stimulation as a new stimulation protocol is promising and needs to be further investigated.

\section{Depression}

Depression is clinical disorder known to result from a disruption of brain neurochemistry (Akhtar et al., 2016). It is a neuronal abnormality characterized by disorders of mood, cognitive function, and neurovegatative functions and has a wide range of causes (Akhtar et al., 2016). Previous neuroimaging studies have demonstrated structural and functional abnormalities in distributed networks of cortical and limbic brain regions including the DLPFC, ventromedial prefrontal cortex, amygdala, hippocampus and subgenual cingulate amongst others (Campbell et al., 2004; Mayberg, 2007; Drevets et al., 2008; Koenigs and Grafman, 2009). Structural and functional abnormalities in these brain regions have also been suggested to be associated with negative affective and cognitive processing bias of depressed patients (Liu S. et al., 2017). Studies have suggested that during the affective processing of depressive individuals, network abnormalities, manifested as enhanced affective processing and decreased cognitive control function, might lead to a more intense experience of negative emotion, inducing depression (Liu S. et al., 2017).

In non-invasive neuromodulation studies, researchers have mostly targeted the left and right DLPFC for depression, as conventional tDCS or traditional rTMS coils were not able to directly and selectively target deeper limbic regions such as the subgenual ACC (Fox et al., 2012a). Deeper limbic regions such as the subgenual ACC have been targeted with invasive neuromodulation, such as deep brain stimulation (e.g., Mayberg et al., 2005; Drevets et al., 2008; Mayberg, 2009; Fox et al., 
2012a) to decrease hyperactivity in this brain region as this has been associated with antidepressant response in depressed patients (Mayberg et al., 2005; Drevets et al., 2008). Regarding more superficial brain areas, researchers have mostly targeted the left DLPFC, but the right DLPFC has also been investigated both in isolation and in a multisite stimulation approach, to suppress depression. The left DLPFC has been found to be hypoactive in patients with depression and an increase in activity is associated with antidepressant response (Fitzgerald et al., 2006b; Koenigs and Grafman, 2009). Besides the hypoactivity found in the left DLPFC, neuroimaging studies have also demonstrated hyperactivity in the right DLPFC in depressive disorder (Bench et al., 1995; Lefaucheur et al., 2014). One of the first FDA-approved clinical uses of rTMS is HF stimulation to the left DLPFC for the treatment of depression (George et al., 1995; Pascual-Leone et al., 1996; O’Reardon et al., 2007; Padberg and George, 2009). In general, the efficacy of HF rTMS to the left DLPFC using a variety of parameters has been established (For review, see Lefaucheur et al., 2014; e.g., Pascual-Leone et al., 1996; Berman et al., 2000; Rossini et al., 2005; Loo et al., 2007; Bretlau et al., 2008; George et al., 2010; Baeken et al., 2013, 2014). LF rTMS to the right DLPFC has also been found effective in several studies (for a review, see Lefaucheur et al., 2014; e.g., Klein et al., 1999; Januel et al., 2006; Fitzgerald et al., 2008; Bares et al., 2009; Aguirre et al., 2011) and there seems to be no difference in antidepressant effect between HF of the left DLPFC and LF rTMS of the right DLPFC (Lefaucheur et al., 2014). For tDCS, earlier studies have mostly targeted the left DLPFC using the electrode montage where the anode is placed over the left DLPFC and the cathode over the right orbitofrontal cortex (for a review, see Lefaucheur et al., 2017; e.g., Boggio et al., 2008a; Loo et al., 2012; Palm et al., 2012; Bennabi et al., 2015). More recently, studies have applied bilateral tDCS, placing the anode electrode over the left DLPFC and the cathode over the right DLPFC instead of the right supraorbital region (e.g., Ferrucci et al., 2009; Brunoni et al., 2013, 2014; Valiengo et al., 2013, 2016, 2017). Several rTMS studies have also investigated a combined modulation of HF left DLPFC rTMS and LF right DLPFC rTMS during the same sessions in the same patients (e.g., Hausmann et al., 2004; Fitzgerald et al., 2006a; Garcia-Toro et al., 2006; McDonald et al., 2006; Pallanti et al., 2010; Blumberger et al., 2012a; Fitzgerald et al., 2012). Lefaucheur et al. (2014), however, do not recommend of using this bilateral rTMS, because of contradictory results across studies.

NIBS has been proposed to normalize the interhemispheric imbalance of neuronal activity by excitatory stimulation of the left DLPFC and inhibitory stimulation over the right DLPFC (Palm et al., 2016; Lefaucheur et al., 2017). According to the "valence hypothesis" the affective processing exhibits hemispheric lateralization, with the right hemisphere specializing in negative emotional processing and the left hemisphere specializing in positive emotional processing (Prete et al., 2015; Liu S. et al., 2017). To further investigate how rTMS of the DLPFC exerts its antidepressant effect, Fox et al. (2012a) have investigated why some left DLPFC rTMS targets are more effective than others by examining differences in functional connectivity of these sites to deeper limbic regions, using resting-state fMRI. They have found that the DLPFC TMS sites with better clinical efficacy were negatively correlated (anticorrelated) with the subgenual ACC. This might suggest a role for intrinsically anticorrelated brain networks in depression implying that the clinical efficacy of focal brain stimulation could be optimized by targeting based on connectivity (Fox et al., 2012a). Depression has indeed been associated by altered intrinsic functional connectivity within and between three intrinsic connectivity networks (ICNs), such as the DMN, the central executive network (CEN) and the salience network ( $\mathrm{SN}$; Manoliu et al., 2013; Anderson et al., 2016; Liu S. et al., 2017). Abnormally increased DMN connectivity has been found in association with depression (Anand et al., 2005; Greicius et al., 2007; Broyd et al., 2009; Sheline et al., 2010; Liston et al., 2014; Kaiser et al., 2015), more specifically related to rumination and deficits in emotion regulation (Sheline et al., 2009; Hamilton et al., 2011). The CEN or the task-positive network, with the DLPFC as a one of the primary nodes, has been found to be hypoconnected in patients with depression (Liston et al., 2014; Kaiser et al., 2015), which may contribute to deficits in memory and attention and other cognitive symptoms in depression (Liston et al., 2014). Furthermore, the SN has shown decreased connectivity in patients with depression, with this aberrant connectivity also demonstrating a significant relationship to depressive symptom severity (Manoliu et al., 2013). Furthermore, these large-scale functional networks have been found to be interacting with each other, with dysfunction in these dynamics associated with depressive symptomatology (Menon, 2011; Manoliu et al., 2013; Anderson et al., 2016). For instance, in depression unusual connectivity has been reported between networks, including abnormal hyperconnectivity between the SN and DMN, and between the DMN and CEN (Manoliu et al., 2013; Liston et al., 2014; Kaiser et al., 2015). In depressed individuals a dominance of DMN over CEN was found which correlated with maladaptive rumination (Hamilton et al., 2011; Wang et al., 2016). Moreover, the aberrant switching between the DMN and CEN in depression has been suggested as a mechanism underlying the preoccupation with self-referential processes related to DMN hyperactivity, and deficits in cognitive functioning, associated with CEN hypoconnectivity (Menon, 2011; Manoliu et al., 2013; Kaiser et al., 2015; Anderson et al., 2016; Wang et al., 2016). The SN has been found to play a causal role in mediating this switching between the DMN and CEN activity (Wang et al., 2016), with specifically the right anterior insula associated with the aberrant DMN/CEN interactions and severity of depressive symptoms (Manoliu et al., 2013). Overall, this evidence demonstrates that dysfunction in connectivity within large-scale functional networks and the interactions in connectivity between these networks are associated with depression (Anderson et al., 2016). Moreover, Liston et al. (2014) have examined the effect of non-invasive neuromodulation in affecting these large-scale brain networks and their relation to treatment response using $\mathrm{HF}(10 \mathrm{~Hz})$ TMS targeting the left DLPFC during a 5-week period (25 sessions). The treatment normalized the depression-related subgenual hyperconnectivity in the DMN, but did not alter the diminished connectivity in the CEN (Liston et al., 2014). TMS also induced an anticorrelated 
connectivity between the DLFPC and the medial prefrontal DMN nodes (Liston et al., 2014).

Another brain region that has been considered a key region in emotion regulation (Mayberg et al., 1999) and that has recently been investigated as a new rTMS target for depression is the dorsomedial prefrontal cortex (DMPFC; i.e., DMN node; Downar and Daskalakis, 2013; Anderson et al., 2016; Liu S. et al., 2017; Liu W. et al., 2017). Studies have suggested that rTMS targeting the DMPFC could show similar antidepressant findings in patients with depression and is a safe procedure (e.g., Downar et al., 2014; Salomons et al., 2014; Bakker et al., 2015). HF rTMS applied bilaterally over the DMPFC demonstrated increased anticorrelation between the DMPFC (DMN node) and the insula (SN node), and increased connectivity with the thalamus (CEN node) to be associated with a greater clinical response in patients with depression (Salomons et al., 2014; Anderson et al., 2016). However more research is needed to investigate the DMPFC as a target for clinical application of rTMS in depression (Liu S. et al., 2017).

\section{ONE STIMULATION TARGET FOR DIFFERENT DISORDERS}

Non-invasive neuromodulation studies stimulating one specific brain region have demonstrated efficacy for various disorders. In this section, we gather evidence to show that tDCS and rTMS over an important brain hub involved in different processes and disorders, i.e., the DLPFC, can have an effect on healthy subjects as well as on various patient populations. The DLPFC is an important brain hub for general cognitive and emotional processing and stimulation of this brain area is expected to influence different processes and disorders.

The DLPFC has been the most common stimulation target in clinical literature to date (Downar et al., 2016). The DLPFC has been the target in several tDCS studies. Anodal tDCS to the left DLPFC has been studied in healthy subjects as well in patient populations. Studies in healthy subjects targeting the DLPFC using anodal tDCS have reported to transiently improve working memory (e.g., Fregni et al., 2005) and attention in healthy subjects (e.g., Nelson et al., 2014). In patient populations, tDCS targeting the DLPFC has been found to show transient improvement in attention in patients with traumatic brain injury (e.g., Kang et al., 2012), in working memory in patients with Parkinson's disease (e.g., Boggio et al., 2006), in recognition memory in patients with Alzheimer disease (e.g., Ferrucci et al., 2008), in signs of consciousness in patients in a minimally conscious state (e.g., Angelakis et al., 2014; Thibaut et al., 2014, 2017), in mood in patients with depression (e.g., Blumberger et al., 2012b; Loo et al., 2012; Palm et al., 2012), in auditory hallucinations in patients with schizophrenia (e.g., Brunelin et al., 2012) and in craving in substance abusers (e.g., Boggio et al., 2008b, 2009; Fregni et al., 2008). In rTMS studies, the left and right DLPFC have been the target for LF and HF rTMS studies in depression (e.g., George et al., 2010; Triggs et al., 2010; Ray et al., 2011; Baeken et al., 2013, 2014), post-traumatic stress disorder (e.g., Boggio et al., 2010; Watts et al., 2012), panic disorder (e.g., Mantovani et al., 2013), schizophrenia targeting the negative symptoms (e.g., Rollnik et al., 2000; Hajak et al., 2004; Cordes et al., 2010; Prikryl et al., 2013) and in addiction disorders-more specifically food (e.g., Van den Eynde et al., 2010; Barth et al., 2011), alcohol (e.g., Mishra et al., 2010) and cigarettes (e.g., Li et al., 2013; Prikryl et al., 2014).

These studies support the idea that neuromodulation of important brain hubs such as the DLPFC can have an effect on different processes in healthy subjects and on different disorders through its influence on a functional brain network. In patient populations, studies have associated the (dorsolateral) prefrontal cortex with top-down prefrontal control, important in various disorder including tinnitus (Mitchell et al., 2005), substance use disorder (Bradshaw et al., 2017), pain (Martinez et al., 2017), depression (Ochsner and Gross, 2005) and schizophrenia (Mayer et al., 2015). A recent study by Tik et al. (2017) further demonstrated that stimulation of the left DLFPC modulates ACC connectivity in a specific meso-cortico-limbic network, which might explain the treatment mechanism of psychiatric disorders such as depression.

In healthy subjects, studies have focused on cognitive processing. Tremblay et al. (2014) have concluded that prefrontal tDCS targeting the DLFPC has the potential to modulate numerous cognitive functions simultaneously and that the effect of prefrontal tDCS on a given task is probably associated with the extensive modulation of a wide range of cognitive functions. Using resting state functional connectivity, Krishnamurthy et al. (2015) further suggest that tDCS might prime not just the underlying neocortex, but an extended network that can be recruited according to the task demands. According to the authors, these priming effects might explain why similar montages have yielded tDCS-induced effects across multiple motor and cognitive tasks. Luft et al. (2014) suggest that the DLPFC can be considered a functional "flexible hub". A flexible hub is defined as a brain region that rapidly updates its pattern of global functional connectivity according to the task demands (Luft et al., 2014). These flexible hubs play an important role in switching from one state to the other in order to attend to the necessary task demands (Luft et al., 2014). Thus, there are indications that modulating an important brain hub using neuromodulation can influence a whole brain network.

\section{DISCUSSION}

The brain is a complex network and, therefore, studying and treating brain disorders using non-invasive neuromodulation techniques should be approached as a network phenomenon. In this article, we have presented support from different perspectives to demonstrate that non-invasive neuromodulation techniques, such as rTMS and tDCS modulate brain networks rather than just local stimulation targets. Evidence for the effectiveness of different stimulation targets for one disorder as well as evidence for the effectiveness of one stimulation target for different disorders indicates an underlying neural network for disorders and, thus, points to the idea of network stimulation as a novel stimulation protocol. Moreover, studies 
assessing network connectivity on both the macro and micro levels help describe and explain the distributed processing of neuromodulation in functional brain networks. However, there are some limitations to connectivity assessed with NIBS in a clinical context. First, NIBS, e.g., tDCS and rTMS, stimulates neuronal tissues exogenously and artificially and, thus, connectivity revealed by stimulation may be different than connectivity present under more physiological conditions (Fox et al., 2012b). Also, connectivity measured with rTMS alone can only be assessed in the cortex with a clear output effect, such as the motor or the visual cortex; investigating other brain areas or the connectivity between other structures needs the addition of neuroimaging techniques. For tDCS, as the electrical currents delivered by tDCS are not strong enough to fire an action potential, connectivity can only be assessed using neuroimaging techniques. Further, non-invasive measurements of human brain activity are highly susceptible to noise and remote changes observed in response to NIBS by

\section{REFERENCES}

Abraham, W. C. (2008). Metaplasticity: tuning synapses and networks for plasticity. Nat. Rev. Neurosci. 9:387. doi: 10.1038/nrn2356

Abraham, W. C., and Bear, M. F. (1996). Metaplasticity: the plasticity of synaptic plasticity. Trends Neurosci. 19, 126-130. doi: 10.1016/s0166-2236(96)80018-X

Aguirre, I., Carretero, B., Ibarra, O., Kuhalainen, J., Martínez, J., Ferrer, A., et al. (2011). Age predicts low-frequency transcranial magnetic stimulation efficacy in major depression. J. Affect. Disord. 130, 466-469. doi: 10.1016/j.jad. 2010.10.038

Akhtar, H., Bukhari, F., Nazir, M., Anwar, M. N., and Shahzad, A. (2016). Therapeutic efficacy of neurostimulation for depression: techniques, current modalities, and future challenges. Neurosci. Bull. 32, 115-126. doi: 10.1007/s12264-015-0009-2

Alon, G., Roys, S. R., Gullapalli, R. P., and Greenspan, J. D. (2011). Non-invasive electrical stimulation of the brain (ESB) modifies the resting-state network connectivity of the primary motor cortex: a proof of concept fMRI study. Brain Res. 1403, 37-44. doi: 10.1016/j.brainres.2011.06.013

Anand, A., Li, Y., Wang, Y., Wu, J., Gao, S., Bukhari, L., et al. (2005). Activity and connectivity of brain mood regulating circuit in depression: a functional magnetic resonance study. Biol. Psychiatry 57, 1079-1088. doi: 10.1016/j. biopsych.2005.02.021

Anderson, R. J., Hoy, K. E., Daskalakis, Z. J., and Fitzgerald, P. B. (2016). Repetitive transcranial magnetic stimulation for treatment resistant depression: re-establishing connections. Clin. Neurophysiol. 127, 3394-3405. doi: 10.1016/j. clinph.2016.08.015

Andoh, J., and Paus, T. (2011). Combining functional neuroimaging with off-line brain stimulation: modulation of task-related activity in language areas. J. Cogn. Neurosci. 23, 349-361. doi: 10.1162/jocn.2010.21449

Angelakis, E., Liouta, E., Andreadis, N., Korfias, S., Ktonas, P., Stranjalis, G., et al. (2014). Transcranial direct current stimulation effects in disorders of consciousness. Arch. Phys. Med. Rehabil. 95, 283-289. doi: 10.1016/j.apmr. 2013.09.002

Arnold, W., Bartenstein, P., Oestreicher, E., Romer, W., and Schwaiger, M. (1996). Focal metabolic activation in the predominant left auditory cortex in patients suffering from tinnitus: a PET study with $[18 \mathrm{~F}]$ deoxyglucose. ORL J. Otorhinolaryngol. Relat. Spec. 58, 195-199. doi: 10.1159/000 276835

Avenanti, A., Coccia, M., Ladavas, E., Provinciali, L., and Ceravolo, M. G. (2012). Low-frequency rTMS promotes use-dependent motor plasticity in chronic stroke: a randomized trial. Neurology 78, 256-264. doi: 10.1212/WNL. 0b013e3182436558

Bachtiar, V., Near, J., Johansen-Berg, H., and Stagg, C. J. (2015). Modulation of GABA and resting state functional connectivity by transcranial direct current stimulation. Elife 4:e08789. doi: 10.7554/eLife.08789 neuroimaging techniques and could reflect other factors besides propagation of NIBS activity along the cortical connection creating interpretative ambiguity (Fox et al., 2012b). These factors could include behavioral and cognitive consequences of NIBS leading to changes in brain activity, or neural adaptation to the NIBS (Fox et al., 2012b). Combining non-invasive neuromodulation with neuroimaging and brain network theories will further elucidate the impact of neuromodulation on brain connectivity and will assist in the development of stimulation protocols to target brain networks, and not just brain regions.

\section{AUTHOR CONTRIBUTIONS}

WTT prepared the manuscript draft with important intellectual input from DDR, JH and SV. All authors approved the final manuscript.

Baeken, C., Marinazzo, D., Wu, G. R., Van Schuerbeek, P., De Mey, J., Marchetti, I. et al. (2014). Accelerated HF-rTMS in treatment-resistant unipolar depression: insights from subgenual anterior cingulate functional connectivity. World J. Biol. Psychiatry 15, 286-297. doi: 10.3109/15622975.2013. 872295

Baeken, C., Vanderhasselt, M. A., Remue, J., Herremans, S., Vanderbruggen, N., Zeeuws, D., et al. (2013). Intensive HF-rTMS treatment in refractory medication-resistant unipolar depressed patients. J. Affect. Disord. 151, 625-631. doi: 10.1016/j.jad.2013.07.008

Bakker, N., Shahab, S., Giacobbe, P., Blumberger, D. M., Daskalakis, Z. J., Kennedy, S. H., et al. (2015). rTMS of the dorsomedial prefrontal cortex for major depression: safety, tolerability, effectiveness, and outcome predictors for $10 \mathrm{~Hz}$ versus intermittent theta-burst stimulation. Brain Stimul. 8, 208-215. doi: 10.1016/j.brs.2014.11.002

Bares, M., Kopecek, M., Novak, T., Stopkova, P., Sos, P., Kozeny, J., et al. (2009). Low frequency $(1-\mathrm{Hz})$, right prefrontal repetitive transcranial magnetic stimulation (rTMS) compared with venlafaxine ER in the treatment of resistant depression: a double-blind, single-centre, randomized study. J. Affect. Disord. 118, 94-100. doi: 10.1016/j.jad.2009.01.032

Barth, K. S., Rydin-Gray, S., Kose, S., Borckardt, J. J., O’Neil, P. M., Shaw, D., et al. (2011). Food cravings and the effects of left prefrontal repetitive transcranial magnetic stimulation using an improved sham condition. Front. Psychiatry 2:9. doi: 10.3389/fpsyt.2011.00009

Bench, C. J., Frackowiak, R. S., and Dolan, R. J. (1995). Changes in regional cerebral blood flow on recovery from depression. Psychol. Med. 25, 247-261. doi: 10.1017/s0033291700036151

Bennabi, D., Nicolier, M., Monnin, J., Tio, G., Pazart, L., Vandel, P., et al. (2015). Pilot study of feasibility of the effect of treatment with tDCS in patients suffering from treatment-resistant depression treated with escitalopram. Clin. Neurophysiol. 126, 1185-1189. doi: 10.1016/j.clinph.2014.09.026

Benninger, D. H., Lomarev, M., Lopez, G., Wassermann, E. M., Li, X., Considine, E., et al. (2010). Transcranial direct current stimulation for the treatment of Parkinson's disease. J. Neurol. Neurosurg. Psychiatry 81, 1105-1111. doi: 10.1136/jnnp.2009.202556

Berlim, M. T., Van den Eynde, F., and Daskalakis, Z. J. (2013). Clinical utility of transcranial direct current stimulation (tDCS) for treating major depression: a systematic review and meta-analysis of randomized, double-blind and sham-controlled trials. J. Psychiatr. Res. 47, 1-7. doi: 10.1016/j.jpsychires.2012. 09.025

Berman, R. M., Narasimhan, M., Sanacora, G., Miano, A. P., Hoffman, R. E., $\mathrm{Hu}, \mathrm{X}$. S., et al. (2000). A randomized clinical trial of repetitive transcranial magnetic stimulation in the treatment of major depression. Biol. Psychiatry 47, 332-337. doi: 10.1016/S0006-3223(99)00243-7

Blumberger, D. M., Mulsant, B. H., Fitzgerald, P. B., Rajji, T. K., Ravindran, A. V., Young, L. T., et al. (2012a). A randomized double-blind sham-controlled 
comparison of unilateral and bilateral repetitive transcranial magnetic stimulation for treatment-resistant major depression. World J. Biol. Psychiatry 13, 423-435. doi: 10.3109/15622975.2011.579163

Blumberger, D. M., Tran, L. C., Fitzgerald, P. B., Hoy, K. E., and Daskalakis, Z. J. (2012b). A randomized double-blind sham-controlled study of transcranial direct current stimulation for treatment-resistant major depression. Front. Psychiatry 3:74. doi: 10.3389/fpsyt.2012.00074

Bocci, T., Caleo, M., Tognazzi, S., Francini, N., Briscese, L., Maffei, L., et al. (2014). Evidence for metaplasticity in the human visual cortex. J. Neural. Transm. (Vienna) 121, 221-231. doi: 10.1007/s00702-013-1104-z

Boggio, P. S., Ferrucci, R., Rigonatti, S. P., Covre, P., Nitsche, M., PascualLeone, A., et al. (2006). Effects of transcranial direct current stimulation on working memory in patients with Parkinson's disease. J. Neurol. Sci. 249, 31-38. doi: 10.1016/j.jns.2006.05.062

Boggio, P. S., Liguori, P., Sultani, N., Rezende, L., Fecteau, S., and Fregni, F. (2009). Cumulative priming effects of cortical stimulation on smoking cue-induced craving. Neurosci. Lett. 463, 82-86. doi: 10.1016/j.neulet.2009. 07.041

Boggio, P. S., Rigonatti, S. P., Ribeiro, R. B., Myczkowski, M. L., Nitsche, M. A., Pascual-Leone, A., et al. (2008a). A randomized, double-blind clinical trial on the efficacy of cortical direct current stimulation for the treatment of major depression. Int. J. Neuropsychopharmacol. 11, 249-254. doi: $10.1017 / \mathrm{s} 1461145707007833$

Boggio, P. S., Sultani, N., Fecteau, S., Merabet, L., Mecca, T., Pascual-Leone, A., et al. (2008b). Prefrontal cortex modulation using transcranial DC stimulation reduces alcohol craving: a double-blind, sham-controlled study. Drug Alcohol Depend. 92, 55-60. doi: 10.1016/j.drugalcdep.2007.06.011

Boggio, P. S., Rocha, M., Oliveira, M. O., Fecteau, S., Cohen, R. B., Campanhã, C., et al. (2010). Noninvasive brain stimulation with high-frequency and low-intensity repetitive transcranial magnetic stimulation treatment for posttraumatic stress disorder. J. Clin. Psychiatry 71, 992-999. doi: 10.4088/JCP. 08m04638blu

Borckardt, J. J., Bikson, M., Frohman, H., Reeves, S. T., Datta, A., Bansal, V., et al. (2012). A pilot study of the tolerability and effects of high-definition transcranial direct current stimulation (HD-tDCS) on pain perception. J. Pain 13, 112-120. doi: 10.1016/j.jpain.2011.07.001

Bradshaw, S. D., Shumway, S. T., Dsauza, C. M., Morris, N., and Hayes, N. D. (2017). Hope, coping skills, and the prefrontal cortex in alcohol use disorder recovery. Am. J. Drug Alcohol Abuse 43, 591-601. doi: 10.1080/00952990.2017. 1286500

Breakspear, M. (2017). Dynamic models of large-scale brain activity. Nat. Neurosci. 20, 340-352. doi: 10.1038/nn.4497

Bretlau, L. G., Lunde, M., Lindberg, L., Unden, M., Dissing, S., and Bech, P. (2008). Repetitive transcranial magnetic stimulation (rTMS) in combination with escitalopram in patients with treatment-resistant major depression: a double-blind, randomised, sham-controlled trial. Pharmacopsychiatry 41, 41-47. doi: 10.1055/s-2007-993210

Broyd, S. J., Demanuele, C., Debener, S., Helps, S. K., James, C. J., and SonugaBarke, E. J. (2009). Default-mode brain dysfunction in mental disorders: a systematic review. Neurosci. Biobehav. Rev. 33, 279-296. doi: 10.1016/j. neubiorev.2008.09.002

Brunelin, J., Mondino, M., Gassab, L., Haesebaert, F., Gaha, L., SuaudChagny, M. F., et al. (2012). Examining transcranial direct-current stimulation (tDCS) as a treatment for hallucinations in schizophrenia. Am. J. Psychiatry 169, 719-724. doi: 10.1176/appi.ajp.2012.11071091

Brunoni, A. R., Boggio, P. S., De Raedt, R., Benseñor, I. M., Lotufo, P. A., Namur, V., et al. (2014). Cognitive control therapy and transcranial direct current stimulation for depression: a randomized, double-blinded, controlled trial. J. Affect. Disord. 162, 43-49. doi: 10.1016/j.jad.2014.03.026

Brunoni, A. R., Ferrucci, R., Fregni, F., Boggio, P. S., and Priori, A. (2012). Transcranial direct current stimulation for the treatment of major depressive disorder: a summary of preclinical, clinical and translational findings. Prog. Neuropsychopharmacol. Biol. Psychiatry 39, 9-16. doi: 10.1016/j.pnpbp.2012. 05.016

Brunoni, A. R., Valiengo, L., Baccaro, A., Zanáo, T. A., de Oliveira, J. F., Goulart, A., et al. (2013). The sertraline vs. electrical current therapy for treating depression clinical study: results from a factorial, randomized, controlled trial. JAMA Psychiatry 70, 383-391. doi: 10.1001/2013.jamapsychiatry.32
Buldyrev, S. V., Parshani, R., Paul, G., Stanley, H. E., and Havlin, S. (2010). Catastrophic cascade of failures in interdependent networks. Nature 464, 1025-1028. doi: $10.1038 /$ nature 08932

Bullmore, E., and Sporns, O. (2012). The economy of brain network organization. Nat. Rev. Neurosci. 13, 336-349. doi: 10.1038/nrn3214

Campbell, S., Marriott, M., Nahmias, C., and MacQueen, G. M. (2004). Lower hippocampal volume in patients suffering from depression: a meta-analysis. Am. J. Psychiatry 161, 598-607. doi: 10.1176/appi.ajp.161.4.598

Cárdenas-Morales, L., Volz, L. J., Michely, J., Rehme, A. K., Pool, E. M., Nettekoven, C., et al. (2014). Network connectivity and individual responses to brain stimulation in the human motor system. Cereb. Cortex 24, 1697-1707. doi: $10.1093 /$ cercor/bht023

Chib, V. S., Yun, K., Takahashi, H., and Shimojo, S. (2013). Noninvasive remote activation of the ventral midbrain by transcranial direct current stimulation of prefrontal cortex. Transl. Psychiatry 3:e268. doi: 10.1038/tp. 2013.44

Clark, V. P., Coffman, B. A., Trumbo, M. C., and Gasparovic, C. (2011). Transcranial direct current stimulation (tDCS) produces localized and specific alterations in neurochemistry: $\mathrm{A}^{1} \mathrm{H}$ magnetic resonance spectroscopy study. Neurosci. Lett. 500, 67-71. doi: 10.1016/j.neulet.2011.05.244

Cocchi, L., Sale, M. V., Lord, A., Zalesky, A., Breakspear, M., and Mattingley, J. B. (2015). Dissociable effects of local inhibitory and excitatory theta-burst stimulation on large-scale brain dynamics. J. Neurophysiol. 113, 3375-3385. doi: $10.1152 /$ jn. 00850.2014

Cordes, J., Thünker, J., Agelink, M. W., Arends, M., Mobascher, A., Wobrock, T., et al. (2010). Effects of $10 \mathrm{~Hz}$ repetitive transcranial magnetic stimulation (rTMS) on clinical global impression in chronic schizophrenia. Psychiatry Res. 177, 32-36. doi: 10.1016/j.psychres.2009.01.014

Cosentino, G., Fierro, B., Paladino, P., Talamanca, S., Vigneri, S., Palermo, A., et al. (2012). Transcranial direct current stimulation preconditioning modulates the effect of high-frequency repetitive transcranial magnetic stimulation in the human motor cortex. Eur. J. Neurosci. 35, 119-124. doi: 10.1111/j.1460-9568. 2011.07939.x

Crossley, N. A., Mechelli, A., Scott, J., Carletti, F., Fox, P. T., McGuire, P., et al. (2014). The hubs of the human connectome are generally implicated in the anatomy of brain disorders. Brain 137, 2382-2395. doi: 10.1093/brain/ awu132

DaSilva, A. F., Truong, D. Q., DosSantos, M. F., Toback, R. L., Datta, A., and Bikson, M. (2015). State-of-art neuroanatomical target analysis of high-definition and conventional tDCS montages used for migraine and pain control. Front. Neuroanat. 9:89. doi: 10.3389/fnana.2015.00089

Datta, A., Bansal, V., Diaz, J., Patel, J., Reato, D., and Bikson, M. (2009). Gyriprecise head model of transcranial direct current stimulation: improved spatial focality using a ring electrode versus conventional rectangular pad. Brain Stimul. 2, 201.e1-207.e1. doi: 10.1016/j.brs.2009.03.005

Deng, Z.-D., Lisanby, S. H., and Peterchev, A. V. (2014). Coil design considerations for deep transcranial magnetic stimulation. Clin. Neurophysiol. 125, 1202-1212. doi: 10.1016/j.clinph.2013.11.038

De Ridder, D., Song, J. J., and Vanneste, S. (2013). Frontal cortex TMS for tinnitus. Brain Stimul. 6, 355-362. doi: 10.1016/j.brs.2012.07.002

De Ridder, D., and Vanneste, S. (2012). EEG driven tDCS versus bifrontal tDCS for tinnitus. Front. Psychiatry 3:84. doi: 10.3389/fpsyt.2012.00084

De Ridder, D., Vanneste, S., Kovacs, S., Sunaert, S., and Dom, G. (2011). Transient alcohol craving suppression by rTMS of dorsal anterior cingulate: an fMRI and LORETA EEG study. Neurosci. Lett. 496, 5-10. doi: 10.1016/j.neulet.2011. 03.074

De Ridder, D., Vanneste, S., Weisz, N., Londero, A., Schlee, W., Elgoyhen, A. B., et al. (2014). An integrative model of auditory phantom perception: tinnitus as a unified percept of interacting separable subnetworks. Neurosci. Biobehav. Rev. 44, 16-32. doi: 10.1016/j.neubiorev.2013.03.021

Desai, N. S. (2003). Homeostatic plasticity in the CNS: synaptic and intrinsic forms. J. Physiol. Paris 97, 391-402. doi: 10.1016/j.jphysparis.2004.01.005

Di Lazzaro, V., Pilato, F., Saturno, E., Oliviero, A., Dileone, M., Mazzone, P., et al. (2005). Theta-burst repetitive transcranial magnetic stimulation suppresses specific excitatory circuits in the human motor cortex. J. Physiol. 565, 945-950. doi: 10.1113/jphysiol.2005.087288

Ding, L., Shou, G., Yuan, H., Urbano, D., and Cha, Y. H. (2014). Lasting modulation effects of rTMS on neural activity and connectivity as 
revealed by resting-state EEG. IEEE Trans. Biomed. Eng. 61, 2070-2080. doi: 10.1109/TBME.2014.2313575

Dmochowski, J. P., Datta, A., Bikson, M., Su, Y., and Parra, L. C. (2011). Optimized multi-electrode stimulation increases focality and intensity at target. J. Neural Eng. 8:046011. doi: 10.1088/1741-2560/8/4/ 046011

Donnell, A., D Nascimento, T., Lawrence, M., Gupta, V., Zieba, T., Truong, D. Q., et al. (2015). High-definition and non-invasive brain modulation of pain and motor dysfunction in chronic TMD. Brain Stimul. 8, 1085-1092. doi: 10.1016/j. brs.2015.06.008

Douglas, Z. H., Maniscalco, B., Hallett, M., Wassermann, E. M., and He, B. J. (2015). Modulating conscious movement intention by noninvasive brain stimulation and the underlying neural mechanisms. J. Neurosci. 35, 7239-7255. doi: 10.1523/JNEUROSCI.4894-14.2015

Downar, J., Blumberger, D. M., and Daskalakis, Z. J. (2016). The neural crossroads of psychiatric illness: an emerging target for brain stimulation. Trends Cogn. Sci. 20, 107-120. doi: 10.1016/j.tics.2015.10.007

Downar, J., and Daskalakis, Z. J. (2013). New targets for rTMS in depression: a review of convergent evidence. Brain Stimul. 6, 231-240. doi: 10.1016/j.brs. 2012.08.006

Downar, J., Geraci, J., Salomons, T. V., Dunlop, K., Wheeler, S., McAndrews, M. P., et al. (2014). Anhedonia and reward-circuit connectivity distinguish nonresponders from responders to dorsomedial prefrontal repetitive transcranial magnetic stimulation in major depression. Biol. Psychiatry 76, 176-185. doi: 10.1016/j.biopsych.2013.10.026

Drevets, W. C., Savitz, J., and Trimble, M. (2008). The subgenual anterior cingulate cortex in mood disorders. CNS Spectr. 13, 663-681. doi: 10.1017/s1092852900013754

Eichhammer, P., Langguth, B., Marienhagen, J., Kleinjung, T., and Hajak, G. (2003). Neuronavigated repetitive transcranial magnetic stimulation in patients with tinnitus: a short case series. Biol. Psychiatry 54, 862-865. doi: 10.1016/s0006-3223(02)01896-6

Faber, M., Vanneste, S., Fregni, F., and De Ridder, D. (2012). Top down prefrontal affective modulation of tinnitus with multiple sessions of tDCS of dorsolateral prefrontal cortex. Brain Stimul. 5, 492-498. doi: 10.1016/j.brs.2011. 09.003

Ferrucci, R., Bortolomasi, M., Vergari, M., Tadini, L., Salvoro, B., Giacopuzzi, M., et al. (2009). Transcranial direct current stimulation in severe, drug-resistant major depression. J. Affect. Disord. 118, 215-219. doi: 10.1016/j.jad.2009. 02.015

Ferrucci, R., Mameli, F., Guidi, I., Mrakic-Sposta, S., Vergari, M., Marceglia, S., et al. (2008). Transcranial direct current stimulation improves recognition memory in Alzheimer disease. Neurology 71, 493-498. doi: 10.1212/01.wnl. 0000317060.43722.a3

Filmer, H. L., Dux, P. E., and Mattingley, J. B. (2014). Applications of transcranial direct current stimulation for understanding brain function. Trends Neurosci. 37, 742-753. doi: 10.1016/j.tins.2014.08.003

Fitzgerald, P. B., Benitez, J., de Castella, A., Daskalakis, Z. J., Brown, T. L., and Kulkarni, J. (2006a). A randomized, controlled trial of sequential bilateral repetitive transcranial magnetic stimulation for treatment-resistant depression. Am. J. Psychiatry 163, 88-94. doi: 10.1176/appi.ajp.163.1.88

Fitzgerald, P. B., Oxley, T. J., Laird, A. R., Kulkarni, J., Egan, G. F., and Daskalakis, Z. J. (2006b). An analysis of functional neuroimaging studies of dorsolateral prefrontal cortical activity in depression. Psychiatry Res. 148, 33-45. doi: 10.1016/j.pscychresns.2006.04.006

Fitzgerald, P. B., Hoy, K. E., Herring, S. E., McQueen, S., Peachey, A. V., Segrave, R. A., et al. (2012). A double blind randomized trial of unilateral left and bilateral prefrontal cortex transcranial magnetic stimulation in treatment resistant major depression. J. Affect. Disord. 139, 193-198. doi: 10.1016/j.jad. 2012.02.017

Fitzgerald, P. B., Hoy, K., McQueen, S., Herring, S., Segrave, R., Been, G., et al. (2008). Priming stimulation enhances the effectiveness of low-frequency right prefrontal cortex transcranial magnetic stimulation in major depression. J. Clin. Psychopharmacol. 28, 52-58. doi: 10.1097/jcp. ob013e3181603f7c

Fornito, A., and Bullmore, E. T. (2015). Connectomics: a new paradigm for understanding brain disease. Eur. Neuropsychopharmacol. 25, 733-748. doi: 10.1016/j.euroneuro.2014.02.011
Fornito, A., Zalesky, A., and Breakspear, M. (2013). Graph analysis of the human connectome: promise, progress, and pitfalls. Neuroimage 80, 426-444. doi: 10.1016/j.neuroimage.2013.04.087

Fornito, A., Zalesky, A., and Breakspear, M. (2015). The connectomics of brain disorders. Nat. Rev. Neurosci. 16, 159-172. doi: 10.1038/nrn3901

Forogh, B., Mirshaki, Z., Raissi, G. R., Shirazi, A., Mansoori, K., and Ahadi, T. (2016). Repeated sessions of transcranial direct current stimulation for treatment of chronic subjective tinnitus: a pilot randomized controlled trial. Neurol. Sci. 37, 253-259. doi: 10.1007/s10072-015-2393-9

Fox, M. D., Buckner, R. L., White, M. P., Greicius, M. D., and Pascual-Leone, A. (2012a). Efficacy of transcranial magnetic stimulation targets for depression is related to intrinsic functional connectivity with the subgenual cingulate. Biol. Psychiatry 72, 595-603. doi: 10.1016/j.biopsych.2012.04.028

Fox, M. D., Halko, M. A., Eldaief, M. C., and Pascual-Leone, A. (2012b). Measuring and manipulating brain connectivity with resting state functional connectivity magnetic resonance imaging (fcMRI) and transcranial magnetic stimulation (TMS). Neuroimage 62, 2232-2243. doi: 10.1016/j.neuroimage.2012. 03.035

Frank, E., Schecklmann, M., Landgrebe, M., Burger, J., Kreuzer, P., Poeppl, T. B., et al. (2012). Treatment of chronic tinnitus with repeated sessions of prefrontal transcranial direct current stimulation: outcomes from an open-label pilot study. J. Neurol. 259, 327-333. doi: 10.1007/s00415-011-6189-4

Fregni, F., Boggio, P. S., Nitsche, M., Bermpohl, F., Antal, A., Feredoes, E., et al. (2005). Anodal transcranial direct current stimulation of prefrontal cortex enhances working memory. Exp. Brain Res. 166, 23-30. doi: 10.1007/s00221005-2334-6

Fregni, F., Liguori, P., Fecteau, S., Nitsche, M. A., Pascual-Leone, A. and Boggio, P. S. (2008). Cortical stimulation of the prefrontal cortex with transcranial direct current stimulation reduces cue-provoked smoking craving: a randomized, sham-controlled study. J. Clin. Psychiatry 69, 32-40. doi: 10.4088 /jcp.v69n0105

Fregni, F., Marcondes, R., Boggio, P. S., Marcolin, M. A., Rigonatti, S. P., Sanchez, T. G., et al. (2006). Transient tinnitus suppression induced by repetitive transcranial magnetic stimulation and transcranial direct current stimulation. Eur. J. Neurol. 13, 996-1001. doi: 10.1111/j.1468-1331.2006. 01414.x

Freitas, C., Mondragón-Llorca, H., and Pascual-Leone, A. (2011). Noninvasive brain stimulation in Alzheimer's disease: systematic review and perspectives for the future. Exp. Gerontol. 46, 611-627. doi: 10.1016/j.exger.2011.04.001

Friston, K. J. (2011). Functional and effective connectivity: a review. Brain Connect. 1, 13-36. doi: 10.1089/brain.2011.0008

Garcia-Toro, M., Salva, J., Daumal, J., Andres, J., Romera, M., Lafau, O., et al. (2006). High $(20-\mathrm{Hz})$ and low $(1-\mathrm{Hz})$ frequency transcranial magnetic stimulation as adjuvant treatment in medication-resistant depression. Psychiatry Res. 146, 53-57. doi: 10.1016/j.pscychresns.2004.08.005

Garin, P., Gilain, C., Van Damme, J. P., de Fays, K., Jamart, J., Ossemann, M., et al. (2011). Short- and long-lasting tinnitus relief induced by transcranial direct current stimulation. J. Neurol. 258, 1940-1948. doi: 10.1007/s00415-011 $-6037-6$

George, M. S., Lisanby, S. H., Avery, D., McDonald, W. M., Durkalski, V., Pavlicova, M., et al. (2010). Daily left prefrontal transcranial magnetic stimulation therapy for major depressive disorder: a sham-controlled randomized trial. Arch. Gen. Psychiatry 67, 507-516. doi: 10.1001/ archgenpsychiatry.2010.46

George, M. S., Wassermann, E. M., Williams, W. A., Callahan, A., Ketter, T. A., Basser, P., et al. (1995). Daily repetitive transcranial magnetic stimulation (rTMS) improves mood in depression. Neuroreport 6, 1853-1856. doi: 10.1097/00001756-199510020-00008

Goodkind, M., Eickhoff, S. B., Oathes, D. J., Jiang, Y., Chang, A., JonesHagata, L. B., et al. (2015). Identification of a common neurobiological substrate for mental illness. JAMA Psychiatry 72, 305-315. doi: 10.1001/ jamapsychiatry.2014.2206

Gratton, C., Lee, T. G., Nomura, E. M., and D'Esposito, M. (2013). The effect of theta-burst TMS on cognitive control networks measured with resting state fMRI. Front. Syst. Neurosci. 7:124. doi: 10.3389/fnsys.2013.00124

Grefkes, C., and Fink, G. R. (2011). Reorganization of cerebral networks after stroke: new insights from neuroimaging with connectivity approaches. Brain 134, 1264-1276. doi: 10.1093/brain/awr033 
Grefkes, C., Nowak, D. A., Wang, L. E., Dafotakis, M., Eickhoff, S. B., and Fink, G. R. (2010). Modulating cortical connectivity in stroke patients by rTMS assessed with fMRI and dynamic causal modeling. Neuroimage 50, 233-242. doi: 10.1016/j.neuroimage.2009.12.029

Greicius, M. D., Flores, B. H., Menon, V., Glover, G. H., Solvason, H. B., Kenna, H., et al. (2007). Resting-state functional connectivity in major depression: abnormally increased contributions from subgenual cingulate cortex and thalamus. Biol. Psychiatry 62, 429-437. doi: 10.1016/j.biopsych.2006.09.020

Guleyupoglu, B., Schestatsky, P., Edwards, D., Fregni, F., and Bikson, M. (2013). Classification of methods in transcranial electrical stimulation (tES) and evolving strategy from historical approaches to contemporary innovations. J. Neurosci. Methods 219, 297-311. doi: 10.1016/j.jneumeth.2013.07.016

Hajak, G., Marienhagen, J., Langguth, B., Werner, S., Binder, H., and Eichhammer, P. (2004). High-frequency repetitive transcranial magnetic stimulation in schizophrenia: a combined treatment and neuroimaging study. Psychol. Med. 34, 1157-1163. doi: 10.1017/s0033291704002338

Hamilton, J. P., Furman, D. J., Chang, C., Thomason, M. E., Dennis, E., and Gotlib, I. H. (2011). Default-mode and task-positive network activity in major depressive disorder: implications for adaptive and maladaptive rumination. Biol. Psychiatry 70, 327-333. doi: 10.1016/j.biopsych.2011.02.003

Hausmann, A., Pascual-Leone, A., Kemmler, G., Rupp, C. I., LechnerSchoner, T., Kramer-Reinstadler, K., et al. (2004). No deterioration of cognitive performance in an aggressive unilateral and bilateral antidepressant rTMS add-on trial. J. Clin. Psychiatry 65, 772-782. doi: 10.4088/jcp.v65 n0608

Hayward, G., Mehta, M. A., Harmer, C., Spinks, T. J., Grasby, P. M., and Goodwin, G. M. (2007). Exploring the physiological effects of double-cone coil TMS over the medial frontal cortex on the anterior cingulate cortex: an $\mathrm{H}_{2}{ }^{15} \mathrm{O}$ PET study. Eur. J. Neurosci. 25, 2224-2233. doi: 10.1111/j.1460-9568. 2007.05430.x

Heimrath, K., Breitling, C., Krauel, K., Heinze, H. J., and Zaehle, T. (2015). Modulation of pre-attentive spectro-temporal feature processing in the human auditory system by HD-tDCS. Eur. J. Neurosci. 41, 1580-1586. doi: 10.1111/ejn. 12908

Horn, D. I., Yu, C., Steiner, J., Buchmann, J., Kaufmann, J., Osoba, A., et al. (2010). Glutamatergic and resting-state functional connectivity correlates of severity in major depression - the role of pregenual anterior cingulate cortex and anterior insula. Front. Syst. Neurosci. 4:33. doi: 10.3389/fnsys.2010.00033

Horvath, J. C., Carter, O., and Forte, J. D. (2014). Transcranial direct current stimulation: five important issues we aren't discussing (but probably should be). Front. Syst. Neurosci. 8:2. doi: 10.3389/fnsys.2014.00002

Huang, X., Gao, J., Buldyrev, S. V., Havlin, S., and Stanley, H. E. (2011). Robustness of interdependent networks under targeted attack. Phys. Rev. E Stat. Nonlin. Soft Matter Phys. 83:065101. doi: 10.1209/0295-5075/103/68005

Huang, Y. Z., Edwards, M. J., Rounis, E., Bhatia, K. P., and Rothwell, J. C. (2005). Theta burst stimulation of the human motor cortex. Neuron 45, 201-206. doi: 10.1016/j.neuron.2004.12.033

Hulme, S. R., Jones, O. D., and Abraham, W. C. (2013). Emerging roles of metaplasticity in behaviour and disease. Trends Neurosci. 36, 353-362. doi: 10.1016/j.tins.2013.03.007

Hunter, M. A., Coffman, B. A., Gasparovic, C., Calhoun, V. D., Trumbo, M. C., and Clark, V. P. (2015). Baseline effects of transcranial direct current stimulation on glutamatergic neurotransmission and large-scale network connectivity. Brain Res. 1594, 92-107. doi: 10.1016/j.brainres.2014.09.066

Hunter, M. A., Coffman, B. A., Trumbo, M. C., and Clark, V. P. (2013). Tracking the neuroplastic changes associated with transcranial direct current stimulation: a push for multimodal imaging. Front. Hum. Neurosci. 7:495. doi: 10.3389/fnhum.2013.00495

Iwabuchi, S. J., Raschke, F., Auer, D. P., Liddle, P. F., Lankappa, S. T., and Palaniyappan, L. (2017). Targeted transcranial theta-burst stimulation alters fronto-insular network and prefrontal GABA. Neuroimage 146, 395-403. doi: 10.1016/j.neuroimage.2016.09.043

Januel, D., Dumortier, G., Verdon, C. M., Stamatiadis, L., Saba, G., Cabaret, W., et al. (2006). A double-blind sham controlled study of right prefrontal repetitive transcranial magnetic stimulation (rTMS): therapeutic and cognitive effect in medication free unipolar depression during 4 weeks. Prog. Neuropsychopharmacol. Biol. Psychiatry 30, 126-130. doi: 10.1016/j.pnpbp. 2005.08.016
Jastreboff, P. J. (1990). Phantom auditory perception (tinnitus): mechanisms of generation and perception. Neurosci. Res. 8, 221-254. doi: 10.1016/01680102(90)90031-9

Ji, G. J., Yu, F., Liao, W., and Wang, K. (2017). Dynamic aftereffects in supplementary motor network following inhibitory transcranial magnetic stimulation protocols. Neuroimage 149, 285-294. doi: 10.1016/j.neuroimage. 2017.01.035

Kaiser, R. H., Andrews-Hanna, J. R., Wager, T. D., and Pizzagalli, D. A. (2015). Large-scale network dysfunction in major depressive disorder: a meta-analysis of resting-state functional connectivity. JAMA Psychiatry 72, 603-611. doi: 10.1001/jamapsychiatry.2015.0071

Kang, E. K., Kim, D. Y., and Paik, N. J. (2012). Transcranial direct current stimulation of the left prefrontal cortex improves attention in patients with traumatic brain injury: a pilot study. J. Rehabil. Med. 44, 346-350. doi: 10.2340/16501977-0947

Kapogiannis, D., Reiter, D. A., Willette, A. A., and Mattson, M. P. (2013). Posteromedial cortex glutamate and GABA predict intrinsic functional connectivity of the default mode network. Neuroimage 64, 112-119. doi: 10.1016/j.neuroimage.2012.09.029

Keeser, D., Meindl, T., Bor, J., Palm, U., Pogarell, O., Mulert, C., et al. (2011a). Prefrontal transcranial direct current stimulation changes connectivity of resting-state networks during fMRI. J. Neurosci. 31, 15284-15293. doi: 10.1523/JNEUROSCI.0542-11.2011

Keeser, D., Padberg, F., Reisinger, E., Pogarell, O., Kirsch, V., Palm, U., et al. (2011b). Prefrontal direct current stimulation modulates resting EEG and event-related potentials in healthy subjects: a standardized low resolution tomography (sLORETA) study. Neuroimage 55, 644-657. doi: 10.1016/j. neuroimage.2010.12.004

Klein, E., Kreinin, I., Chistyakov, A., Koren, D., Mecz, L., Marmur, S., et al. (1999) Therapeutic efficacy of right prefrontal slow repetitive transcranial magnetic stimulation in major depression: a double-blind controlled study. Arch. Gen. Psychiatry 56, 315-320. doi: 10.1001/archpsyc.56.4.315

Kleinjung, T., Eichhammer, P., Landgrebe, M., Sand, P., Hajak, G., Steffens, T., et al. (2008). Combined temporal and prefrontal transcranial magnetic stimulation for tinnitus treatment: a pilot study. Otolaryngol. Head Neck Surg. 138, 497-501. doi: 10.1016/j.otohns.2007.12.022

Koenigs, M., and Grafman, J. (2009). The functional neuroanatomy of depression: distinct roles for ventromedial and dorsolateral prefrontal cortex. Behav. Brain Res. 201, 239-243. doi: 10.1016/j.bbr.2009.03.004

Kreuzer, P. M., Lehner, A., Schlee, W., Vielsmeier, V., Schecklmann, M., Poeppl, T. B., et al. (2015). Combined rTMS treatment targeting the anterior cingulate and the temporal cortex for the treatment of chronic tinnitus. Sci. Rep. 5:18028. doi: 10.1038/srep18028

Krishnamurthy, V., Gopinath, K., Brown, G. S., and Hampstead, B. M. (2015). Resting-state fMRI reveals enhanced functional connectivity in spatial navigation networks after transcranial direct current stimulation. Neurosci. Lett. 604, 80-85. doi: 10.1016/j.neulet.2015.07.042

Kunze, T., Hunold, A., Haueisen, J., Jirsa, V., and Spiegler, A. (2016). Transcranial direct current stimulation changes resting state functional connectivity: a largescale brain network modeling study. Neuroimage 140, 174-187. doi: 10.1016/j. neuroimage.2016.02.015

Lang, N., Siebner, H. R., Ernst, D., Nitsche, M. A., Paulus, W., Lemon, R. N., et al. (2004). Preconditioning with transcranial direct current stimulation sensitizes the motor cortex to rapid-rate transcranial magnetic stimulation and controls the direction of after-effects. Biol. Psychiatry 56, 634-639. doi: 10.1016/j. biopsych.2004.07.017

Langguth, B., Eichhammer, P., Wiegand, R., Marienhegen, J., Maenner, P., Jacob, P., et al. (2003). Neuronavigated rTMS in a patient with chronic tinnitus. Effects of 4 weeks treatment. Neuroreport 14, 977-980. doi: 10.1097/01.WNR. 0000068897.39523 .41

Langguth, B., Schecklmann, M., Lehner, A., Landgrebe, M., Poeppl, T. B., Kreuzer, P. M., et al. (2012). Neuroimaging and neuromodulation: complementary approaches for identifying the neuronal correlates of tinnitus. Front. Syst. Neurosci. 6:15. doi: 10.3389/fnsys.2012.00015

Lefaucheur, J. P., André-Obadia, N., Antal, A., Ayache, S. S., Baeken, C., Benninger, D. H., et al. (2014). Evidence-based guidelines on the therapeutic use of repetitive transcranial magnetic stimulation (rTMS). Clin. Neurophysiol. 125, 2150-2206. doi: 10.1016/j.clinph.2014.05.021 
Lefaucheur, J. P., Antal, A., Ayache, S. S., Benninger, D. H., Brunelin, J., Cogiamanian, F., et al. (2017). Evidence-based guidelines on the therapeutic use of transcranial direct current stimulation (tDCS). Clin. Neurophysiol. 128, 56-92. doi: 10.1016/j.clinph.2016.10.087

Lehner, A., Schecklmann, M., Poeppl, T. B., Kreuzer, P. M., Vielsmeier, V., Rupprecht, R., et al. (2013). Multisite rTMS for the treatment of chronic tinnitus: stimulation of the cortical tinnitus network-a pilot study. Brain Topogr. 26, 501-510. doi: 10.1007/s10548-012-0268-4

Li, X., Hartwell, K. J., Owens, M., Lematty, T., Borckardt, J. J., Hanlon, C. A., et al. (2013). Repetitive transcranial magnetic stimulation of the dorsolateral prefrontal cortex reduces nicotine cue craving. Biol. Psychiatry 73, 714-720. doi: 10.1016/j.biopsych.2013.01.003

Liebetanz, D., Nitsche, M. A., Tergau, F., and Paulus, W. (2002). Pharmacological approach to the mechanisms of transcranial DC-stimulation-induced after-effects of human motor cortex excitability. Brain 125, 2238-2247. doi: 10.1093/brain/awf238

Liston, C., Chen, A. C., Zebley, B. D., Drysdale, A. T., Gordon, R., Leuchter, B., et al. (2014). Default mode network mechanisms of transcranial magnetic stimulation in depression. Biol. Psychiatry 76, 517-526. doi: 10.1016/j.biopsych. 2014.01.023

Liu, W., Leng, Y. S., Zou, X. H., Cheng, Z. Q., Yang, W., and Li, B. J. (2017). Affective processing in non-invasive brain stimulation over prefrontal cortex. Front. Hum. Neurosci. 11:439. doi: 10.3389/fnhum.2017.00439

Liu, S., Sheng, J., Li, B., and Zhang, X. (2017). Recent advances in non-invasive brain stimulation for major depressive disorder. Front. Hum. Neurosci. 11:526. doi: 10.3389/fnhum.2017.00526

Lockwood, A. H., Salvi, R. J., Coad, M. L., Towsley, M. L., Wack, D. S., and Murphy, B. W. (1998). The functional neuroanatomy of tinnitus: evidence for limbic system links and neural plasticity. Neurology 50, 114-120. doi: 10.1212/WNL.50.1.114

Loo, C. K., Alonzo, A., Martin, D., Mitchell, P. B., Galvez, V., and Sachdev, P. (2012). Transcranial direct current stimulation for depression: 3-week, randomised, sham-controlled trial. Br. J. Psychiatry 200, 52-59. doi: 10.1192/bjp.bp.111.097634

Loo, C. K., Mitchell, P. B., McFarquhar, T. F., Malhi, G. S., and Sachdev, P. S. (2007). A sham-controlled trial of the efficacy and safety of twice-daily rTMS in major depression. Psychol. Med. 37, 341-349. doi: 10.1017/s003329170 6009597

Lorenz, I., Müller, N., Schlee, W., Hartmann, T., and Weisz, N. (2009). Loss of $\alpha$ power is related to increased $\gamma$ synchronization-A marker of reduced inhibition in tinnitus? Neurosci. Lett. 453, 225-228. doi: 10.1016/j.neulet.2009.02.028

Luft, C. D., Pereda, E., Banissy, M. J., and Bhattacharya, J. (2014). Best of both worlds: promise of combining brain stimulation and brain connectome. Front. Syst. Neurosci. 8:132. doi: 10.3389/fnsys.2014.00132

Mancini, M., Mastropasqua, C., Bonnì, S., Ponzo, V., Cercignani, M., Conforto, S., et al. (2017). Theta burst stimulation of the precuneus modulates resting state connectivity in the left temporal pole. Brain Topogr. 30, 312-319. doi: 10.1007/s10548-017-0559-x

Manoliu, A., Meng, C., Brandl, F., Doll, A., Tahmasian, M., Scherr, M., et al. (2013). Insular dysfunction within the salience network is associated with severity of symptoms and aberrant inter-network connectivity in major depressive disorder. Front. Hum. Neurosci. 7:930. doi: 10.3389/fnhum.2013.00930

Mantovani, A., Aly, M., Dagan, Y., Allart, A., and Lisanby, S. H. (2013). Randomized sham controlled trial of repetitive transcranial magnetic stimulation to the dorsolateral prefrontal cortex for the treatment of panic disorder with comorbid major depression. J. Affect. Disord. 144, 153-159. doi: 10.1016/j.jad.2012.05.038

Marangolo, P., Fiori, V., Calpagnano, M. A., Campana, S., Razzano, C., Caltagirone, C., et al. (2013). tDCS over the left inferior frontal cortex improves speech production in aphasia. Front. Hum. Neurosci. 7:539. doi: 10.3389/fnhum.2013.00539

Martinez, E., Lin, H. H., Zhou, H., Dale, J., Liu, K., and Wang, J. (2017). Corticostriatal regulation of acute pain. Front. Cell. Neurosci. 11:146. doi: 10.3389/fncel.2017.00146

Mastropasqua, C., Bozzali, M., Ponzo, V., Giulietti, G., Caltagirone, C., Cercignani, M., et al. (2014). Network based statistical analysis detects changes induced by continuous theta-burst stimulation on brain activity at rest. Front. Psychiatry 5:97. doi: 10.3389/fpsyt.2014.00097
Mayberg, H. S. (2007). Defining the neural circuitry of depression: toward a new nosology with therapeutic implications. Biol. Psychiatry 61, 729-730. doi: 10.1016/j.biopsych.2007.01.013

Mayberg, H. S. (2009). Targeted electrode-based modulation of neural circuits for depression. J. Clin. Invest. 119, 717-725. doi: 10.1172/JCI38454

Mayberg, H. S., Liotti, M., Brannan, S. K., McGinnis, S., Mahurin, R. K., Jerabek, P. A., et al. (1999). Reciprocal limbic-cortical function and negative mood: converging PET findings in depression and normal sadness. Am. J. Psychiatry 156, 675-682.

Mayberg, H. S., Lozano, A. M., Voon, V., McNeely, H. E., Seminowicz, D., Hamani, C., et al. (2005). Deep brain stimulation for treatment-resistant depression. Neuron 45, 651-660. doi: 10.1016/j.neuron.2005.02.014

Mayer, A. R., Hanlon, F. M., Teshiba, T. M., Klimaj, S. D., Ling, J. M., Dodd, A. B., et al. (2015). An fMRI study of multimodal selective attention in schizophrenia. Br. J. Psychiatry 207, 420-428. doi: 10.1192/bjp.bp.114.155499

McDonald, W. M., Easley, K., Byrd, E. H., Holtzheimer, P., Tuohy, S., Woodard, J. L., et al. (2006). Combination rapid transcranial magnetic stimulation in treatment refractory depression. Neuropsychiatr. Dis. Treat. 2, 85-94.

Medeiros, L. F., de Souza, I. C., Vidor, L. P., de Souza, A., Deitos, A., Volz, M. S., et al. (2012). Neurobiological effects of transcranial direct current stimulation: a review. Front. Psychiatry 3:110. doi: 10.3389/fpsyt.2012.00110

Meinzer, M., Antonenko, D., Lindenberg, R., Hetzer, S., Ulm, L., Avirame, K., et al. (2012). Electrical brain stimulation improves cognitive performance by modulating functional connectivity and task-specific activation. J. Neurosci. 32, 1859-1866. doi: 10.1523/JNEUROSCI.4812-11.2012

Meinzer, M., Lindenberg, R., Antonenko, D., Flaisch, T., and Floel, A. (2013). Anodal transcranial direct current stimulation temporarily reverses age-associated cognitive decline and functional brain activity changes. J. Neurosci. 33, 12470-12478. doi: 10.1523/JNEUROSCI.5743-12.2013

Menon, V. (2011). Large-scale brain networks and psychopathology: a unifying triple network model. Trends Cogn. Sci. 15, 483-506. doi: 10.1016/j.tics.2011. 08.003

Menon, V. (2013). Developmental pathways to functional brain networks: emerging principles. Trends Cogn. Sci. 17, 627-640. doi: 10.1016/j.tics.2013. 09.015

Mishra, B. R., Nizamie, S. H., Das, B., and Praharaj, S. K. (2010). Efficacy of repetitive transcranial magnetic stimulation in alcohol dependence: a sham-controlled study. Addiction 105, 49-55. doi: 10.1111/j.1360-0443.2009. 02777.x

Mitchell, T. V., Morey, R. A., Inan, S., and Belger, A. (2005). Functional magnetic resonance imaging measure of automatic and controlled auditory processing. Neuroreport 16, 457-461. doi: 10.1097/00001756-200504040-00008

Moisset, X., and Bouhassira, D. (2007). Brain imaging of neuropathic pain. Neuroimage 37, S80-S88. doi: 10.1016/j.neuroimage.2007.03.054

Nelson, J. T., McKinley, R. A., Golob, E. J., Warm, J. S., and Parasuraman, R. (2014). Enhancing vigilance in operators with prefrontal cortex transcranial direct current stimulation (tDCS). Neuroimage 85, 909-917. doi: 10.1016/j. neuroimage.2012.11.061

Nitsche, M. A., Liebetanz, D., Schlitterlau, A., Henschke, U., Fricke, K., Frommann, K., et al. (2004). GABAergic modulation of DC stimulationinduced motor cortex excitability shifts in humans. Eur. J. Neurosci. 19, 2720-2726. doi: 10.1111/j.0953-816x.2004.03398.x

Nitsche, M. A., Seeber, A., Frommann, K., Klein, C. C., Rochford, C., Nitsche, M. S., et al. (2005). Modulating parameters of excitability during and after transcranial direct current stimulation of the human motor cortex. J. Physiol. 568, 291-303. doi: 10.1113/jphysiol.2005.092429

Noh, N. A., Fuggetta, G., and Manganotti, P. (2015). Theta-burst transcranial magnetic stimulation alters the functional topography of the cortical motor network. Malays. J. Med. Sci. 22, 36-44.

Norena, A., Cransac, H., and Chery-Croze, S. (1999). Towards an objectification by classification of tinnitus. Clin. Neurophysiol. 110, 666-675. doi: 10.1016/s13882457(98)00034-0

Ochsner, K. N., and Gross, J. J. (2005). The cognitive control of emotion. Trends Cogn. Sci. 9, 242-249. doi: 10.1016/j.tics.2005.03.010

O’Reardon, J. P., Solvason, H. B., Janicak, P. G., Sampson, S., Isenberg, K. E., Nahas, Z., et al. (2007). Efficacy and safety of transcranial magnetic stimulation in the acute treatment of major depression: a multisite randomized 
controlled trial. Biol. Psychiatry 62, 1208-1216. doi: 10.1016/j.biopsych.2007. 01.018

O'Shea, J., Johansen-Berg, H., Trief, D., Göbel, S., and Rushworth, M. F. (2007). Functionally specific reorganization in human premotor cortex. Neuron 54, 479-490. doi: 10.1016/j.neuron.2007.04.021

Padberg, F., and George, M. S. (2009). Repetitive transcranial magnetic stimulation of the prefrontal cortex in depression. Exp. Neurol. 219, 2-13. doi: 10.1016/j. expneurol.2009.04.020

Pal, N., Maire, R., Stephan, M. A., Herrmann, F. R., and Benninger, D. H. (2015). Transcranial direct current stimulation for the treatment of chronic tinnitus: a randomized controlled study. Brain Stimul. 8, 1101-1107. doi: 10.1016/j.brs. 2015.06.014

Pallanti, S., Bernardi, S., Di Rollo, A., Antonini, S., and Quercioli, L. (2010). Unilateral low frequency versus sequential bilateral repetitive transcranial magnetic stimulation: is simpler better for treatment of resistant depression? Neuroscience 167, 323-328. doi: 10.1016/j.neuroscience.2010.01.063

Palm, U., Hasan, A., Strube, W., and Padberg, F. (2016). tDCS for the treatment of depression: a comprehensive review. Eur. Arch. Psychiatry Clin. Neurosci. 266, 681-694. doi: 10.1007/s00406-016-0674-9

Palm, U., Schiller, C., Fintescu, Z., Obermeier, M., Keeser, D., Reisinger, E., et al. (2012). Transcranial direct current stimulation in treatment resistant depression: a randomized double-blind, placebo-controlled study. Brain Stimul. 5, 242-251. doi: 10.1016/j.brs.2011.08.005

Park, C. H., Chang, W. H., Park, J. Y., Shin, Y. I., Kim, S. T., and Kim, Y. H. (2013). Transcranial direct current stimulation increases resting state interhemispheric connectivity. Neurosci. Lett. 539, 7-10. doi: 10.1016/j.neulet.2013. 01.047

Pascual-Leone, A., Rubio, B., Pallardó, F., and Catalá, M. D. (1996). Rapidrate transcranial magnetic stimulation of left dorsolateral prefrontal cortex in drug-resistant depression. Lancet 348, 233-237. doi: 10.1016/s01406736(96)01219-6

Peña-Gómez, C., Sala-Lonch, R., Junqué, C., Clemente, I. C., Vidal, D., Bargalló, N., et al. (2012). Modulation of large-scale brain networks by transcranial direct current stimulation evidenced by resting-state functional MRI. Brain Stimul. 5, 252-263. doi: 10.1016/j.brs.2011.08.006

Plewnia, C., Bartels, M., and Gerloff, C. (2003). Transient suppression of tinnitus by transcranial magnetic stimulation. Ann. Neurol. 53, 263-266. doi: 10.1002/ana.10468

Polanía, R., Nitsche, M. A., and Paulus, W. (2011a). Modulating functional connectivity patterns and topological functional organization of the human brain with transcranial direct current stimulation. Hum. Brain Mapp. 32, 1236-1249. doi: 10.1002/hbm.21104

Polanía, R., Paulus, W., Antal, A., and Nitsche, M. A. (2011b). Introducing graph theory to track for neuroplastic alterations in the resting human brain: a transcranial direct current stimulation study. Neuroimage 54, 2287-2296. doi: 10.1016/j.neuroimage.2010.09.085

Polanía, R., Paulus, W., and Nitsche, M. A. (2012). Modulating cortico-striatal and thalamo-cortical functional connectivity with transcranial direct current stimulation. Hum. Brain Mapp. 33, 2499-2508. doi: 10.1002/hbm.21380

Prete, G., Laeng, B., Fabri, M., Foschi, N., and Tommasi, L. (2015). Right hemisphere or valence hypothesis, or both? The processing of hybrid faces in the intact and callosotomized brain. Neuropsychologia 68, 94-106. doi: 10.1016/j.neuropsychologia.2015.01.002

Prikryl, R., Ustohal, L., Kucerova, H. P., Kasparek, T., Jarkovsky, J., Hublova, V., et al. (2014). Repetitive transcranial magnetic stimulation reduces cigarette consumption in schizophrenia patients. Prog. Neuropsychopharmacol. Biol. Psychiatry 49, 30-35. doi: 10.1016/j.pnpbp.2013.10.019

Prikryl, R., Ustohal, L., Prikrylova Kucerova, H., Kasparek, T., Venclikova, S., Vrzalova, M., et al. (2013). A detailed analysis of the effect of repetitive transcranial magnetic stimulation on negative symptoms of schizophrenia: a double-blind trial. Schizophr. Res. 149, 167-173. doi: 10.1016/j.schres.2013. 06.015

Radman, T., Ramos, R. L., Brumberg, J. C., and Bikson, M. (2009). Role of cortical cell type and morphology in subthreshold and suprathreshold uniform electric field stimulation in vitro. Brain Stimul. 2, 215-228, 228.e1-228.e3. doi: 10.1016/j.brs.2009.03.007

Rahnev, D., Kok, P., Munneke, M., Bahdo, L., de Lange, F. P., and Lau, H. (2013). Continuous theta burst transcranial magnetic stimulation reduces resting state connectivity between visual areas. J. Neurophysiol. 110, 1811-1821. doi: $10.1152 /$ jn.00209.2013

Rauschecker, J. P., Leaver, A. M., and Mühlau, M. (2010). Tuning out the noise: limbic-auditory interactions in tinnitus. Neuron 66, 819-826. doi: 10.1016/j. neuron.2010.04.032

Ray, S., Nizamie, S. H., Akhtar, S., Praharaj, S. K., Mishra, B. R., and Zia-ulHaq, M. (2011). Efficacy of adjunctive high frequency repetitive transcranial magnetic stimulation of left prefrontal cortex in depression: a randomized sham controlled study. J. Affect. Disord. 128, 153-159. doi: 10.1016/j.jad. 2010.06.027

Rollnik, J. D., Huber, T. J., Mogk, H., Siggelkow, S., Kropp, S., Dengler, R., et al. (2000). High frequency repetitive transcranial magnetic stimulation (rTMS) of the dorsolateral prefrontal cortex in schizophrenic patients. Neuroreport 11, 4013-4015. doi: 10.1097/00001756-200012180-00022

Rossini, D., Magri, L., Lucca, A., Giordani, S., Smeraldi, E., and Zanardi, R. (2005). Does rTMS hasten the response to escitalopram, sertraline, or venlafaxine in patients with major depressive disorder? A double-blind, randomized, sham-controlled trial. J. Clin. Psychiatry 66, 1569-1575. doi: 10.4088/jcp. v66n1212

Rubinov, M., and Bullmore, E. (2013). Schizophrenia and abnormal brain network hubs. Dialogues Clin. Neurosci. 15, 339-349.

Sale, M. V., Mattingley, J. B., Zalesky, A., and Cocchi, L. (2015). Imaging human brain networks to improve the clinical efficacy of non-invasive brain stimulation. Neurosci. Biobehav. Rev. 57, 187-198. doi: 10.1016/j.neubiorev. 2015.09.010

Sale, M. V., Ridding, M. C., and Nordstrom, M. A. (2007). Factors influencing the magnitude and reproducibility of corticomotor excitability changes induced by paired associative stimulation. Exp. Brain Res. 181, 615-626. doi: 10.1007/s00221-007-0960-x

Salomons, T. V., Dunlop, K., Kennedy, S. H., Flint, A., Geraci, J., Giacobbe, P., et al. (2014). Resting-state cortico-thalamic-striatal connectivity predicts response to dorsomedial prefrontal rTMS in major depressive disorder. Neuropsychopharmacology 39, 488-498. doi: 10.1038/npp.2013.222

Sehm, B., Kipping, J., Schäfer, A., Villringer, A., and Ragert, P. (2013). A comparison between Uni- and bilateral tDCS effects on functional connectivity of the human motor cortex. Front. Hum. Neurosci. 7:183. doi: 10.3389/fnhum. 2013.00183

Shafi, M. M., Westover, M. B., Fox, M. D., and Pascual-Leone, A. (2012). Exploration and modulation of brain network interactions with noninvasive brain stimulation in combination with neuroimaging. Eur. J. Neurosci. 35, 805-825. doi: 10.1111/j.1460-9568.2012.08035.x

Shapira-Lichter, I., Oren, N., Jacob, Y., Gruberger, M., and Hendler, T. (2013). Portraying the unique contribution of the default mode network to internally driven mnemonic processes. Proc. Natl. Acad. Sci. U S A 110, 4950-4955. doi: 10.1073/pnas.1209888110

Shekhawat, G. S., Stinear, C. M., and Searchfield, G. D. (2013). Transcranial direct current stimulation intensity and duration effects on tinnitus suppression. Neurorehabil. Neural Repair 27, 164-172. doi: 10.1177/1545968312459908

Shekhawat, G. S., Sundram, F., Bikson, M., Truong, D., De Ridder, D., Stinear, C. M., et al. (2016). Intensity, duration, and location of high-definition transcranial direct current stimulation for tinnitus relief. Neurorehabil. Neural Repair 30, 349-359. doi: 10.1177/1545968315595286

Sheline, Y. I., Barch, D. M., Price, J. L., Rundle, M. M., Vaishnavi, S. N., Snyder, A. Z., et al. (2009). The default mode network and self-referential processes in depression. Proc. Natl. Acad. Sci. U S A 106, 1942-1947. doi: 10.1073/pnas.0812686106

Sheline, Y. I., Price, J. L., Yan, Z., and Mintun, M. A. (2010). Restingstate functional MRI in depression unmasks increased connectivity between networks via the dorsal nexus. Proc. Natl. Acad. Sci. U S A 107, 11020-11025. doi: $10.1073 /$ pnas. 1000446107

Siebner, H. R., Lang, N., Rizzo, V., Nitsche, M. A., Paulus, W., Lemon, R. N., et al. (2004). Preconditioning of low-frequency repetitive transcranial magnetic stimulation with transcranial direct current stimulation: evidence for homeostatic plasticity in the human motor cortex. J. Neurosci. 24, 3379-3385. doi: 10.1523/JNEUROSCI.5316-03.2004

Sporns, O., Tononi, G., and Kötter, R. (2005). The human connectome: a structural description of the human brain. PLoS Comput. Biol. 1:e42. doi: 10.1371/journal. pcbi.0010042 
Stagg, C. J., Bachtiar, V., Amadi, U., Gudberg, C. A., Ilie, A. S., SampaioBaptista, C., et al. (2014). Local GABA concentration is related to network-level resting functional connectivity. Elife 3:e01465. doi: 10.7554/eLife.01465

Stagg, C. J., Best, J. G., Stephenson, M. C., O’Shea, J., Wylezinska, M., Kincses, Z. T., et al. (2009). Polarity-sensitive modulation of cortical neurotransmitters by transcranial stimulation. J. Neurosci. 29, 5202-5206. doi: 10.1523/JNEUROSCI. 4432-08.2009

Stagg, C. J., Lin, R. L., Mezue, M., Segerdahl, A., Kong, Y., Xie, J., et al. (2013). Widespread modulation of cerebral perfusion induced during and after transcranial direct current stimulation applied to the left dorsolateral prefrontal cortex. J. Neurosci. 33, 11425-11431. doi: 10.1523/JNEUROSCI.3887-12.2013

Stagg, C. J., and Nitsche, M. A. (2011). Physiological basis of transcranial direct current stimulation. Neuroscientist 17, 37-53. doi: 10.1177/1073858410386614

Sun, W., Mao, W., Meng, X., Wang, D., Qiao, L., Tao, W., et al. (2012). Lowfrequency repetitive transcranial magnetic stimulation for the treatment of refractory partial epilepsy: a controlled clinical study. Epilepsia 53, 1782-1789. doi: 10.1111/j.1528-1167.2012.03626.x

Thibaut, A., Bruno, M. A., Ledoux, D., Demertzi, A., and Laureys, S. (2014). tDCS in patients with disorders of consciousness: sham-controlled randomized double-blind study. Neurology 82, 1112-1118. doi: 10.1212/WNL. 0000000000000260

Thibaut, A., Wannez, S., Donneau, A. F., Chatelle, C., Gosseries, O., Bruno, M. A., et al. (2017). Controlled clinical trial of repeated prefrontal tDCS in patients with chronic minimally conscious state. Brain Inj. 31, 466-474. doi: 10.1080/02699052.2016.1274776

Thiebaut de Schotten, M., Dell'Acqua, F., Ratiu, P., Leslie, A., Howells, H., Cabanis, E., et al. (2015). From phineas gage and monsieur leborgne to H.M.: revisiting disconnection syndromes. Cereb. Cortex 25, 4812-4827. doi: $10.1093 /$ cercor/bhv173

Thirugnanasambandam, N., Grundey, J., Adam, K., Drees, A., Skwirba, A. C., Lang, N., et al. (2011). Nicotinergic impact on focal and non-focal neuroplasticity induced by non-invasive brain stimulation in non-smoking humans. Neuropsychopharmacology 36, 879-886. doi: 10.1038/npp.2010.227

Tik, M., Hoffmann, A., Sladky, R., Tomova, L., Hummer, A., Navarro de Lara, L., et al. (2017). Towards understanding rTMS mechanism of action: stimulation of the DLPFC causes network-specific increase in functional connectivity. Neuroimage 162, 289-296. doi: 10.1016/j.neuroimage.2017.09.022

Tremblay, S., Lepage, J. F., Latulipe-Loiselle, A., Fregni, F., Pascual-Leone, A., and Théoret, H. (2014). The uncertain outcome of prefrontal tDCS. Brain Stimul. 7, 773-783. doi: 10.1016/j.brs.2014.10.003

Triggs, W. J., Ricciuti, N., Ward, H. E., Cheng, J., Bowers, D., Goodman, W. K., et al. (2010). Right and left dorsolateral pre-frontal rTMS treatment of refractory depression: a randomized, sham-controlled trial. Psychiatry Res. 178, 467-474. doi: 10.1016/j.psychres.2010.05.009

Valchev, N., Ćurčić-Blake, B., Renken, R. J., Avenanti, A., Keysers, C., Gazzola, V., et al. (2015). cTBS delivered to the left somatosensory cortex changes its functional connectivity during rest. Neuroimage 114, 386-397. doi: 10.1016/j. neuroimage.2015.04.017

Valiengo, L., Benseñor, I. M., Goulart, A. C., de Oliveira, J. F., Zanao, T. A., Boggio, P. S., et al. (2013). The sertraline versus electrical current therapy for treating depression clinical study (select-TDCS): results of the crossover and follow-up phases. Depress. Anxiety 30, 646-653. doi: 10.1002/da.22079

Valiengo, L., Casati, R., Bolognini, N., Lotufo, P. A., Bensenor, I. M., Goulart, A. C., et al. (2016). Transcranial direct current stimulation for the treatment of post-stroke depression in aphasic patients: a case series. Neurocase 22, 225-228. doi: 10.1080/13554794.2015.1130231

Valiengo, L. C., Goulart, A. C., de Oliveira, J. F., Benseñor, I. M., Lotufo, P. A., and Brunoni, A. R. (2017). Transcranial direct current stimulation for the treatment of post-stroke depression: results from a randomised, sham-controlled, doubleblinded trial. J. Neurol. Neurosurg. Psychiatry 88, 170-175. doi: 10.1136/jnnp2016-314075

Van den Eynde, F., Claudino, A. M., Mogg, A., Horrell, L., Stahl, D., Ribeiro, W., et al. (2010). Repetitive transcranial magnetic stimulation reduces cue-induced food craving in bulimic disorders. Biol. Psychiatry 67, 793-795. doi: 10.1016/j. biopsych.2009.11.023 van den Heuvel, M. P., and Sporns, O. (2011). Rich-club organization of the human connectome. J. Neurosci. 31, 15775-15786. doi: 10.1523/JNEUROSCI.3539 $-11.2011$

van den Heuvel, M. P., and Sporns, O. (2013a). An anatomical substrate for integration among functional networks in human cortex. J. Neurosci. 33, 14489-14500. doi: 10.1523/JNEUROSCI.2128-13.2013

van den Heuvel, M. P., and Sporns, O. (2013b). Network hubs in the human brain. Trends Cogn. Sci. 17, 683-696. doi: 10.1016/j.tics.2013.09.012

Van Doren, J., Langguth, B., and Schecklmann, M. (2014). Electroencephalographic effects of transcranial random noise stimulation in the auditory cortex. Brain Stimul. 7, 807-812. doi: 10.1016/j.brs.2014. 08.007

Vanneste, S., and De Ridder, D. (2011). Bifrontal transcranial direct current stimulation modulates tinnitus intensity and tinnitus-distress-related brain activity. Eur. J. Neurosci. 34, 605-614. doi: 10.1111/j.1460-9568.2011.07778.x

Vanneste, S., and De Ridder, D. (2012). The auditory and non-auditory brain areas involved in tinnitus. An emergent property of multiple parallel overlapping subnetworks. Front. Syst. Neurosci. 6:31. doi: 10.3389/fnsys.2012.00031

Vanneste, S., Fregni, F., and De Ridder, D. (2013a). Head-to-head comparison of transcranial random noise stimulation, transcranial AC stimulation and transcranial DC stimulation for tinnitus. Front. Psychiatry 4:158. doi: 10.3389/fpsyt.2013.00158

Vanneste, S., Walsh, V., Van De Heyning, P., and De Ridder, D. (2013b). Comparing immediate transient tinnitus suppression using tACS and tDCS: a placebo-controlled study. Exp. Brain Res. 226, 25-31. doi: 10.1007/s00221-0133406-7

Vanneste, S., Plazier, M., Ost, J., van der Loo, E., Van de Heyning, P., and De Ridder, D. (2010). Bilateral dorsolateral prefrontal cortex modulation for tinnitus by transcranial direct current stimulation: a preliminary clinical study. Exp. Brain Res. 202, 779-785. doi: 10.1007/s00221-010-2183-9

Villamar, M. F., Volz, M. S., Bikson, M., Datta, A., Dasilva, A. F., and Fregni, F. (2013a). Technique and considerations in the use of $4 \times 1$ ring high-definition transcranial direct current stimulation (HD-tDCS). J. Vis. Exp. 77:e50309. doi: $10.3791 / 50309$

Villamar, M. F., Wivatvongvana, P., Patumanond, J., Bikson, M., Truong, D. Q., Datta, A., et al. (2013b). Focal modulation of the primary motor cortex in fibromyalgia using $4 \times 1$-ring high-definition transcranial direct current stimulation (HD-tDCS): immediate and delayed analgesic effects of cathodal and anodal stimulation. J. Pain 14, 371-383. doi: 10.1016/j.jpain.2012. 12.007

Wang, X., Öngür, D., Auerbach, R. P., and Yao, S. (2016). Cognitive vulnerability to major depression: view from the intrinsic network and crossnetwork interactions. Harv. Rev. Psychiatry 24, 188-201. doi: 10.1097/HRP. 0000000000000081

Watts, B. V., Landon, B., Groft, A., and Young-Xu, Y. (2012). A sham controlled study of repetitive transcranial magnetic stimulation for posttraumatic stress disorder. Brain Stimul. 5, 38-43. doi: 10.1016/j.brs.2011.02.002

Weber, M. J., Messing, S. B., Rao, H., Detre, J. A., and Thompson-Schill, S. L. (2014). Prefrontal transcranial direct current stimulation alters activation and connectivity in cortical and subcortical reward systems: a tDCS-fMRI study. Hum. Brain Mapp. 35, 3673-3686. doi: 10.1002/hbm.22429

Zyss, T. (2010). Transcranial direct current stimulation and related techniques in treatment of psychiatric disorders. Psychiatr. Pol. 44, 505-518.

Conflict of Interest Statement: The authors declare that the research was conducted in the absence of any commercial or financial relationships that could be construed as a potential conflict of interest.

Copyright (C) 2018 To, De Ridder, Hart and Vanneste. This is an open-access article distributed under the terms of the Creative Commons Attribution License (CC BY). The use, distribution or reproduction in other forums is permitted, provided the original author(s) and the copyright owner are credited and that the original publication in this journal is cited, in accordance with accepted academic practice. No use, distribution or reproduction is permitted which does not comply with these terms. 